\title{
1 DRP1-mediated regulation of mitochondrial dynamics 2 determines the apoptotic response upon embryonic 3 differentiation.
}

4 Barbara Pernaute ${ }^{1,2, *}$, Juan Miguel Sánchez Nieto ${ }^{1,3^{*}}$, Salvador Pérez-Montero ${ }^{1, *}$, Aida 5 di Gregorio ${ }^{1}$, Ana Lima ${ }^{1}$, Katerina Lawlor ${ }^{1}$, Sarah Bowling ${ }^{1}$, Gianmaria Liccardi ${ }^{4}$, 6 Alejandra Tomás, ${ }^{5}$ Pascal Meier ${ }^{4}$, Guy A. Rutter ${ }^{5}$, Ivana Barbaric ${ }^{6}$ and Tristan A. $7 \quad$ Rodríguez $^{1 \S}$

$8{ }^{1}$ National Heart and Lung Institute, Imperial College London, Hammersmith Hospital

9 Campus, Du Cane Road, London W12 0NN, United Kingdom.

$10{ }^{2}$ Current address: Centre for Genomic Regulation (CRG), The Barcelona Institute of Science

11 and Technology, Dr. Aiguader 88, Barcelona 08003, Spain

$12{ }^{3}$ Current address: Cell and Gene Therapy Catapult. $12^{\text {th }}$ Floor Tower Wing, Guy's Hospital,

13 Great Maze Pond, London SE1 9RT. United Kingdom.

$14{ }^{4}$ The Breast Cancer Now Toby Robins Research Centre, The Institute of Cancer Research,

15 London, UK

$16{ }^{5}$ Department of Metabolism, Digestion and Reproduction, Imperial College London, London,

17 United Kingdom,

$18{ }^{6}$ Department of Biomedical Science, The University of Sheffield, Western Bank, Sheffield

19 S10 2TN, United Kingdom

$21 \quad$ Equal contributing first authors.

$22 \S$ Author for correspondence tristan.rodriguez@imperial.ac.uk 


\section{Abstract}

24 The changes that drive differentiation create a large potential for the emergence of abnormal 25 cells that need to be removed before they contribute to further development or the germline.

26 This removal is in part achieved by cells becoming hypersensitive to death upon exit of naïve 27 pluripotency. What causes this change in apoptotic response is unknown. Here we identify 28 that it is controlled by the regulator of mitochondrial dynamics DRP1. We show that in 29 mouse, naïve pluripotent cells have fragmented mitochondria due to high DRP1-mediated 30 fission, but upon differentiation, DRP1 activity decreases, inducing mitochondria to fuse and 31 form complex networks. We demonstrate that this decrease in DRP1 activity lowers the 32 apoptotic threshold, as mutation of DRP1 increases the sensitivity to cell death and its over33 expression protects against apoptosis. Together, our findings highlight how regulation of 34 mitochondrial dynamics allows cells to adapt their apoptotic response to the changing 35 environment of differentiation. 


\section{Introduction}

38 The appropriate regulation of apoptosis during embryonic development is essential for maintaining the right balance between the elimination of suboptimal cells and the availability of sufficient cell numbers to sustain embryo growth ${ }^{1}$. The maintenance of this balance is most acutely evident in the lead up to gastrulation, when the mouse epiblast not only undergoes the process of germ layer specification, but also displays a significant expansion in cell numbers, from 150 at E5.5 to 15,000 cells at E7.5 ${ }^{2}$. This rapid proliferation of cells is concomitant with the substantial cellular changes that accompany gastrulation, most notably extinction of the pluripotency network and activation of differentiation genes in a lineage-specific manner. Any abnormal or mis-specified cell, which cannot perform these changes appropriately, needs to be removed from the embryo to prevent them from contributing to further development or the germline, which is also specified around E6.5 $5^{3}$. Hence, a wave of cell death takes place in the mouse embryo at E6.5, most likely reflecting the elimination of these abnormal cells ${ }^{4,5}$.

One way by which the embryo facilitates the elimination of aberrant cells is through lowering the apoptotic threshold as it proceeds from a pre-implantation to an early post-implantation stage of development. Indeed, whilst low doses of UV irradiation do not induce apoptosis in mouse pre-implantation embryos, these same low doses lead to a strong apoptotic response in the post-implantation epiblast ${ }^{6}$. Similarly, although cells with a range of genetic defects, including chromosome fragmentation or chromosome mis-segregation survive preimplantation development, they are efficiently eliminated by apoptosis during early postimplantation development ${ }^{7-10}$. Subsequently, it has been proposed that the post-implantation epiblast is primed for death, and therefore hyper-responsive to apoptotic signals ${ }^{6,11}$.

The mitochondrial apoptotic pathway is tightly regulated by a balance between pro- and antiapoptotic factors belonging to the BCL-2 family. The binding of the anti-apoptotic proteins (e.g BCL-2, BCL-XL, A1 or MCL-1) to their pro-apoptotic BCL-2 family counterparts (e.g.

63 BIM, BID, PUMA, NOXA or BAD) prevents the induction of apoptosis. When this balance 64 is lost, BIM or BID bind to the apoptotic effector molecules BAX and BAK, which induce 65 mitochondrial outer membrane permeabilization (MOMP) and caspase activation ${ }^{12}$. We have previously shown that, in the post-implantation epiblast, this balance is at least in part maintained by microRNAs (miRNAs) of the miR-20, miR-92, and miR-302 families. These miRNAs target Bim $(B c l 2 l 11)$ and maintain it in a state that is poised for activation ${ }^{11}$. However, it is worth noting that mutation of Bim does not prevent the endogenous wave of cell death occurring during early mouse development, suggesting that other factors must be contributing to making the epiblast hypersensitive to death signals ${ }^{11}$.

The balance between mitochondrial fusion and fission, termed mitochondrial dynamics, has emerged over the last few years as an important regulator of the apoptotic response. Mitochondrial dynamics are facilitated by proteins such as MFN1, MFN2 and OPA1, that promote fusion and DRP1 or MFF, that promote fission ${ }^{13-15}$. During cell death, remodelling 
77 cytochrome $\mathrm{C}$ and subsequent apoptosis. DRP1-induced mitochondrial fragmentation is also

78 coupled to the later stages of apoptosis ${ }^{19-22}$. Finally, MFN1-induced fusion has been shown to 79 promote a mitochondrial size that is permissive for BAX function ${ }^{23}$. Therefore, the regulators

80 of mitochondrial dynamics play a variety of different roles in the apoptotic process. In the 81 developing embryo, mitochondrial shape undergoes substantial changes around the time of 82 implantation $^{24}$. During pre-implantation stages mitochondria are round and have a low cristae 83 density $^{25}$, but later in development mitochondria elongate and cristae density increases ${ }^{24}$. 84 These observations raise the possibility that changes in mitochondrial dynamics may play 85 roles in regulating the changes in apoptotic threshold that occur during early development.

86 Here we have identified that a change in mitochondrial dynamics increases the sensitivity to cell death signals in cells of the post-implantation epiblast. For this, we combined experiments in the embryo, with studies using embryonic stem cells (ESCs), which capture the naïve pluripotent state found in the pre-implantation epiblast, and in epiblast stem cells (EpiSCs), which resemble the primed pluripotent post-implantation epiblast ${ }^{26}$. We find that although primed pluripotent cells show high mitochondrial apoptotic priming, their increased sensitivity to cell death is not due to differential expression of members of the BCL-2 proand anti-apoptotic family. In contrast, we observed that decreased mitochondrial fission correlates with the readiness of cells to undergo apoptosis. Furthermore, we demonstrate that manipulating DRP1 activity is sufficient to change the apoptotic threshold of pluripotent cells. Together, these results demonstrate that changes in mitochondrial dynamics influence the apoptotic priming status of cells and contribute to the elimination of aberrant cells during early embryonic development.

\section{Results}

100 Pluripotent cells become hypersensitive to cell death upon exit of naïve pluripotency.

101 We set out firstly to characterise the apoptotic sensitivity of the different pluripotent cell 102 types found during early mouse development. Previous studies have shown that whilst cells 103 from the pre-implantation embryo are relatively resistant to low doses of UV radiation, the 104 early post-implantation cells respond to the same low doses of UV by undergoing apoptosis ${ }^{6}$. 105 In line with these findings, we observed no overt apoptosis in E3.5 mouse embryos treated 106 with the DNA damage-inducing drug etoposide for $1.5 \mathrm{~h}$, but we detected a strong apoptotic 107 response when the etoposide treatment was applied to E6.5 embryos (Supplementary Figure 108 1A-B).

109 We next tested whether the observed differential sensitivity to apoptosis is also apparent in 110 ESCs and EpiSCs, the two in vitro counterparts of the pre- and post-implantation epiblast ${ }^{26}$. 111 We have previously shown that loss of miRNAs leads to an upregulation of BIM expression 112 and a consequent induction of apoptosis in EpiSCs ${ }^{11}$. To test if miRNAs also regulate 113 apoptosis in ESCs, we induced Dicer deletion in Dicer $^{F x / F x}$ ESCs by tamoxifen 114 administration, as we had previously done for EpiSCs ${ }^{11}$. This led to miRNA depletion 115 (Supplementary Figure 1C) and an up-regulation of BIM expression similar to that found in 116 EpiSCs (Figure 1A). However, in contrast to what was seen in EpiSCs, the increase in BIM 
117 expression did not cause apoptosis in ESCs (Figure 1B-C and Supplementary Figure 1D).

118 This suggests that mouse ESCs are more resistant to apoptosis than EpiSCs.

119 Importantly, we found that the differential apoptotic response of ESCs and EpiSCs was also 120 evident upon exposing these cells to different sources of stress. Induction of oxidative stress 121 with $1 \mu \mathrm{M}$ sodium arsenite for 16 hours produced a 3-fold increase in Annexin $\mathrm{V}$ positive 122 ESCs, but a 7-fold increase in Annexin V positive EpiSCs (Figure 1D). Similarly, induction 123 of endoplasmic reticulum (ER) stress with increasing doses of thapsigargin for 16 hours led 124 to a small change in the basal levels of cleaved Caspase 3 in ESCs, whereas the same 125 treatment induced a robust apoptotic response in EpiSCs (Figure 1E). Overall, these results 126 indicate that EpiSCs exhibit an increased sensitivity to apoptosis when compared to ESCs, 127 thus recapitulating the features observed in their in vivo counterparts.

\section{The mitochondrial apoptotic pathway is primed for cell death in primed pluripotent} 129 stem cells.

130 We next addressed if the differences in apoptotic response between naïve and primed 131 pluripotent cells were reflected at the level of the mitochondrial apoptotic pathway. This 132 pathway is regulated by the relative expression of pro-apoptotic and anti-apoptotic BCL-2 133 family members ${ }^{12}$. This balance can be artificially changed, for example by inhibiting BCL134 2/XL activity with BH3 mimetics such as ABT-737. We observed that 24h treatment of 135 EpiSCs with ABT-737 led to a strong increase in the percentage of cells displaying (1) 136 MOMP (Supplementary Figure 1E), (2) Annexin V positivity (Figure 2A) and (3) cleaved 137 caspase-3 expression (Figure 2B). In contrast, treatment with ABT-737 failed to induce 138 apoptosis in ESCs, even at the highest concentration used (Figure 2A-B and Supplementary 139 Figure 1E-F). These findings are strengthened by the observation that ABT-737 leads to 140 cytochrome $\mathrm{C}$ release from the mitochondria in EpiSCs, but not ESCs (Figure 2B). To test 141 the physiological significance of these observations, we treated pre- and post-implantation 142 mouse embryos with $2 \mu \mathrm{M}$ ABT-737 for $1.5 \mathrm{~h}$. Similar to what we observed in vitro, we found 143 that this treatment induced apoptosis in epiblast cells of E6.5 embryos, but not in inner cell 144 mass cells of E3.5 embryos (Figure 2C and Supplementary Figure 1F).

145 Next, we determined whether the enhanced sensitivity of EpiSCs to BCL2/BCLXL inhibition 146 was dependent on BIM. For this we transfected previously tested siRNAs targeting Bim ${ }^{11}$ 147 into EpiSCs and treated them with ABT-737. We observed that this was sufficient to suppress 148 the apoptotic response induced by BCL2/BCL-XL inhibition (Figure 2D), further 149 highlighting the importance of BIM for the apoptotic response of EpiSCs. Given that the 150 differences in apoptotic response between ESCs and EpiSCs appear to be mediated by the 151 mitochondrial apoptotic pathway, we measured the level of mitochondrial apoptotic priming 152 of these cells by analysing the kinetics of mitochondrial membrane depolarization induced by 153 BIM and BID BH3 peptides ${ }^{27}$. We observed that the depolarization of the mitochondrial 154 membrane potential was significantly more pronounced in EpiSCs than in ESCs for both 155 these peptides (Figure 2E). Together these results indicate that primed pluripotent cells have 
156 a lower apoptotic threshold than naïve cells due to enhanced sensitivity of the mitochondrial 157 pathway.

158 The relative expression of pro- and anti-apoptotic BCL2 family members does not 159 explain the different apoptotic threshold of ESCs and EpiSCs.

160 To address if differences in the relative expression of pro- and anti-apoptotic BCL2 family 161 members underpin the different apoptotic response of ESCs and EpiSCs, we compared the 162 expression of these proteins in each cell type. We observed no significant difference in the 163 expression of the pro-apoptotic activator proteins PUMA, BIM, BID or BAD in either whole 164 cell or mitochondrial extracts between these two cell types (Figure 3A). Similarly, we did not 165 observe any difference in the expression of the pro-apoptotic effector proteins BAX or BAK 166 (Figure 3B). In contrast, when anti-apoptotic proteins were analysed, we found little 167 difference in the expression of BCL-XL, MCL1 or A1, but significantly higher expression of 168 BCL-2 in EpiSCs, in both whole cell and mitochondrial extracts (Figure 3C). Whilst a higher 169 expression of anti-apoptotic protein expression may seem counterintuitive given the 170 enhanced sensitivity to death of EpiSCs, this elevated BCL-2 expression is likely part of the 171 adaptation of these cells to their low apoptotic threshold ${ }^{28}$.

172 To determine if the balance of expression of pro- and anti-apoptotic factors changes 173 differently in ESCs and EpiSCs upon induction of apoptosis, we analysed the expression of 174 key BCL-2 family members after treating these cells with $5 \mu \mathrm{M}$ ABT-737 for 24 hours. In 175 these experiments we analysed the expression of the anti-apoptotic BCL-2 family members 176 as well as BIM expression, as BIM is required for the apoptotic response to ABT-737 (Figure 177 2D). However, we observed no significant change in the levels of expression of BCL-2, 178 BCL-XL, A1, MCL-1 or BIM between ABT-737 treated samples and controls in either whole 179 cell or mitochondrial extracts of ESCs or EpiSCs (Supplementary Figure 2A-B). This 180 suggests that the induction of apoptosis does not significantly shift the balance of pro- and 181 anti-apoptotic BCL-2 family expression. Together, these results indicate that the relative 182 expression of BCL-2 family proteins is not the cause of the higher sensitivity to apoptosis of 183 primed pluripotent cells.

184 High levels of mitochondrial fission are observed in naïve pluripotent cells when 185 compared to primed cells.

186 Mitochondrial dynamics have been suggested as a mechanism that contributes to the 187 regulation of the mitochondrial apoptotic threshold ${ }^{13}$. The mitochondria of ESCs and pre188 implantation embryos have been shown to be rounded ${ }^{25}$ and to elongate upon 189 differentiation ${ }^{29}, 30$. Analysis of mitochondrial morphology in ESCs revealed rounded 190 doughnut shaped mitochondria in agreement with previous studies ${ }^{30}$. In contrast, the 191 mitochondria of EpiSCs were more elongated (Figure 4A). Importantly, these differences 192 were also seen in vivo, with E3.5 inner cell mass cells and E4.5 epiblast cells having rounded 193 mitochondria and the mitochondria of E6.5 epiblast cells being elongated and forming 194 networks (Figure 4B). 
195 The differences in mitochondrial shape between naïve and primed pluripotent cells suggest 196 that these cell types have different mitochondrial dynamics. We therefore studied the 197 expression of fusion and fission regulators in ESCs and EpiSCs. While we observed no 198 difference in the expression of the fusion regulators MFN1, MFN2 or OPA1 between these 199 cell types, we noticed that ESCs had significantly higher levels of total DRP1 and p-DRP1 200 (S616), a phosphorylation event that induces fission ${ }^{31}$ (Figure 4C-D). This indicates that 201 naïve pluripotent cells have higher fission activity. To determine if the higher p-DRP1 202 expression is responsible for the rounded mitochondrial shape of ESCs, we knocked-out 203 Drp1 by CRISPR-Cas9. Although Drp1 $1^{-/}$ESCs remained pluripotent, their mitochondria 204 became elongated and hyperfused (Figure 5A-B and Supplementary Figure 3A). These 205 results indicate that DRP1-induced mitochondrial fission is required for the fragmented 206 mitochondrial shape observed in ESCs.

207 DRP1 plays a key role in determining the apoptotic threshold of naïve and primed 208 pluripotent cells.

209 To evaluate whether DRP1 levels determine the apoptotic threshold of pluripotent cells we 210 first analysed the effects of loss of Drpl. In the first instance we compared the apoptotic 211 response of wild-type and $D r p 1^{-/}$ESCs to ER stress or oxidative stress. We observed that a 212 significantly higher proportion of $\mathrm{Drpl}^{-/}$cells treated with thapsigargin or sodium arsenite 213 show MOMP (Figure 5C). We next analysed the kinetics of MOMP in response to BH3 214 peptides and observed that both BIM and BID BH3 peptides were more efficient at inducing 215 mitochondrial outer membrane depolarization in $\operatorname{Drpl}^{-1}$ ESCs than wild-type cells (Figure 216 5E). This indicates that loss of Drpl is sufficient to lower the apoptotic threshold of ESCs 217 (Figure 2E). Importantly, this change in sensitivity to cell death induction was unlikely to be 218 a secondary consequence of a disruption of the metabolism of mutant cells, as Drp1 $1^{-/}$ESCs 219 did not show decreased glycolysis or oxidative phosphorylation rates when compared to 220 wild-type cells. Instead, mutant cells displayed an increased spare respiratory capacity, an 221 increased response to pyruvate and an increased glycolytic rate (Supplementary Figure 3B222 E), suggesting that mitochondrial fission enhances the bioenergetic rate of pluripotent cells.

223 DRP1 has been shown to be involved in the remodelling of the mitochondrial cristae and the 224 subsequent release of cytochrome $\mathrm{C}$ into the cytoplasm ${ }^{17}, 18$, an event downstream of MOMP. 225 We therefore analysed if cytochrome $\mathrm{C}$ release is compromised in $\operatorname{Drpl}^{-/} \mathrm{ESCs}$. We 226 observed that Drpl $^{-/}$ESCs displayed lower levels of cytoplasmic cytochrome C upon 227 thapsigargin treatment (Supplementary Figure 3F). This suggests that DRP1 is likely to play 228 at least two roles in the apoptotic response in pluripotent cells. First, it impedes the pro229 apoptotic roles of BCL-2 family members and second it promotes cytochrome C release by 230 helping the remodelling of the mitochondrial cristae.

231 Given the roles of DRP1 in determining the apoptotic response of ESCs, we next wanted to 232 analyse its importance during ESCs differentiation. During differentiation p-DRP1 levels 233 decrease (Figure 4C) and therefore we analysed the effects of Drpl overexpression (Drpl ${ }^{O E}$ ) 234 in differentiating ESCs. We have previously shown that culturing ESCs for 3 days in N2B27 
235 leads to a post-implantation epiblast-like state ${ }^{32}$. We therefore cultured Drpl ${ }^{O E}$ cells for 3

236 days in N2B27 and found that this led to sustained DRP1 expression and increase in p-DRP1

237 levels (Supplementary Figure 4A-C and Supplementary Figure 5A-D). Importantly, this

238 increase in p-DRP1 did not prevent exit of naïve pluripotency but induced mitochondria to

239 adopt a more rounded morphology (Figure 6A-C and Supplementary Figure 5A-B). We

240 therefore tested the effect on the apoptotic response of a sustained increase in p-DRP1 during

241 the onset of ESC differentiation. We found that after 3 days in differentiation conditions,

$242 \operatorname{Drpl}^{O E}$ cells displayed not only lower basal cleaved caspase 3 levels (Figure 6D and

243 Supplementary Figure 5A-C), but also significantly reduced levels of cleaved Caspase 3 in

244 response to treatment with thapsigargin, sodium arsenite or ABT-737 (Figure 6E-G and

245 Supplementary Figure 5A,C-D). Together with our findings in Drp1 ${ }^{-/}$ESCs, these results

246 suggest that the decrease in DRP1 and p-DRP1 levels observed during exit of pluripotency

247 play an important role in sensitising cells to apoptosis.

\section{Discussion}

249 The removal of aberrant cells during development is important to prevent them from 250 contributing to further development and the germline. To facilitate this elimination cells 251 become hypersensitive to apoptosis during the onset of differentiation ${ }^{6,11,33,34}$. Here we 252 show that DRP1 regulates apoptotic priming in these cells via influencing mitochondrial 253 dynamics. We show that upon exit of naïve pluripotency mitochondria form complex 254 networks due to a decrease in mitochondrial fission induced by a loss of DRP1 255 phosphorylation. We also demonstrate that this loss of DRP1 activity changes the apoptotic 256 threshold, as deletion of Drpl facilitates the early stages of apoptosis and its over-expression 257 protects against apoptosis. Together, these findings highlight the pivotal role that DRP1 plays 258 in modulating the response to cell death during differentiation.

259 When considering how DRP1 levels might affect the apoptotic response, a number of 260 possibilities arise. In the first instance, it has been shown that mitochondrial shape affects the 261 ability of BAX to permeabilise the outer mitochondria membrane. It was found that 262 mitochondrial fusion establishes a mitochondrial size that is permissive to the function of 263 pro-apoptotic BCL2 family members and that mitochondrial hyper-fragmentation inhibited 264 the ability of BAX to associate with and permeabilise the outer mitochondria membrane ${ }^{23}$. In 265 line with this possibility, the rounded and fragmented shape of the mitochondria of naive 266 pluripotent cells due to the high levels of fission induced by DRP1 would help explain the 267 high apoptotic threshold of these cells.

268 An alternative, but not mutually exclusive, possibility that could explain how DRP1 affects 269 the apoptotic response, is by modulating the entry of calcium into the mitochondria. It is well 270 known that the degree of contact between the endoplasmic reticulum (ER) and mitochondria 271 has profound implications for the function of each of these organelles ${ }^{13,35}$. For example entry 272 of calcium into the mitochondria is thought to be regulated by ER-mitochondria contacts. 273 Moreover, ER-mitochondria contact sites are thought to be a site of reactive oxygen species 
274 (ROS) signalling, which also affects calcium signals delivery to the mitochondria ${ }^{36}$. We

275 observe that both mutation of DRP1 or its overexpression changes the apoptotic response to

276 thapsigargin, a drug that increases calcium levels in the cytoplasm, and to sodium arsenite,

277 which induces oxidative stress. Therefore, our results raise the possibility that by regulating

278 mitochondrial shape, DRP1 levels control the degree of contact between the ER and

279 mitochondria, with the formation of elongated mitochondrial networks induced by decreased

280 fission during differentiation favouring increased mitochondria ER-contacts and in this way

281 facilitating calcium entry into the mitochondria.

282 It is worth highlighting that the involvement of DRP1 in the early apoptotic events involving 283 permeabilization of the mitochondrial membrane are separable from the downstream release 284 of cytochrome $\mathrm{C}$, which others have shown and we also find is promoted by DRP $1^{17,18,37}$. In 285 principle, this would represent two opposing roles for DRP1: slowing mitochondrial 286 membrane permeabilization, but facilitating cytochrome $\mathrm{C}$ release. One way to reconcile 287 these potentially opposing roles is that either each one requires different levels of DRP1, with 288 the release cytochrome $\mathrm{C}$ being possible with just baseline DRP1 levels. Alternatively, 289 different post-translational modifications of DRP1 may lead to altered function. Indeed, 290 DRP1 has been shown to be phosphorylated at 3 different sites, as well as being S291 nitrolisilated, O-GlcNAcylated, SUMOylated and ubiquitinated ${ }^{13}$, 38 and each of these 292 modifications are associated with different roles of DRP1. We have only analysed S616 293 phosphorylation and found it to be lost during ESC differentiation, but to gain a more in 294 depth understanding of the roles of DRP1 in the apoptotic response, it would be important to 295 analyse how a broader range of post-transcriptional modifications differ between the naïve 296 and primed pluripotent states.

297 Our results contrast with the roles that others have identified for mitochondrial dynamics in 298 pluripotency. For example over-expression of $M f f$, a fission regulator, inhibits the expression 299 of neural markers during pluripotent stem cell differentiation ${ }^{39}$, suggesting that inhibiting 300 mitochondrial elongation disrupts differentiation. Similarly, mutation of Mtch2, a potential 301 fusion regulator, delays exit from naïve pluripotency ${ }^{40}$, leading to the suggestion that 302 mitochondrial fusion promotes differentiation. In contrast to this we find that Drpl null 303 ESCs, which have hyper-fused mitochondria, show normal pluripotency gene expression and 304 over-expression of Drpl does not affect exit from naïve pluripotency. What these results 305 suggest, is that mitochondrial elongation per se is not linked to exit of pluripotency, but 306 rather it is likely that other mitochondrial processes regulated by Mff and Mtch2 have an 307 impact on the onset of differentiation.

308 Finally, our work has implications that transcend early mammalian development as DRP1 has 309 been shown to play roles in tumour progression. For example, in glioblastoma DRP1 310 activation is correlated with poor prognosis. Mechanistically this is explained as brain tumour 311 initiating cells have fragmented mitochondria and require high p-DRP1 levels for their 312 survival $^{41}$. Similarly, in pancreatic ductal adenocarcinomas, oncogenic Ras mutations induces 313 mitochondrial fragmentation. Reversion of this phenotype by knock-down of Drpl inhibits 314 tumour growth ${ }^{42,}{ }^{43}$, further highlighting the potential importance of DRP1 for tumour 
315 progression. Our findings that in embryonic stem cells DRP1 promotes cell survival, raises

316 the possibility that part of this oncogenic role is through the regulation of the apoptotic 317 response. Understanding the players downstream of DRP1 will therefore likely open new 318 avenues for our understanding of transformation.

\section{Acknowledgements}

320 We would like to Massimo Signore and Juan Pedro Martinez Barbera for critical discussion. 321 We thank Stephen Rothery for guidance and advice with confocal microscopy. Gratitude also 322 goes to James Elliot for performing cell sorting. Research in the Tristan Rodriguez lab was 323 supported by an MRC project grant (MR/N009371/1) and by the British Heart Foundation 324 centre for research excellence. Barbara Pernaute was supported by an EMBO Long Term 325 Fellowship (1340-2010) and a Marie Curie Intra-European Fellowship (FP7-PEOPLE-2010326 IEF $n^{\circ}$ 273884). Salvador Perez Montero has been a recipient of an EMBO long-term 327 Fellowship (846-2015) and a Marie Curie Intra-European Fellowship (H2020-MSCA-IF$3282015 \mathrm{n}^{\mathbf{0}}$ 709010). Work in the Meier lab is funded by Breast Cancer Now (CTR-QR14-007). 329 Guy A. Rutter was supported by Wellcome Trust Senior Investigator (WT098424AIA ) and 330 Investigator (212625/Z/18/Z) Awards, MRC Programme grants (MR/R022259/1, $331 \mathrm{MR} / \mathrm{J} 0003042 / 1, \quad \mathrm{MR} / \mathrm{L} 020149 / 1)$ and Experimental Challenge Grant (DIVA, $332 \mathrm{MR} / \mathrm{L} 02036 \mathrm{X} / 1)$, MRC (MR/N00275X/1), and Diabetes UK (BDA/11/0004210, 333 BDA/15/0005275, BDA 16/0005485) grants. This project has received funding from the 334 European Union via the Innovative Medicines Initiative 2 Joint Undertaking under grant 335 agreement No 115881 (RHAPSODY) to Guy A. Rutter. We acknowledge NHS funding to 336 the NIHR Biomedical Research Centre. Alejandra Tomás is funded by the MRC project grant 337 MR/R010676/1. 


\section{Figure Legends}

340 Figure 1. The apoptotic threshold decreases upon pluripotent stem cell differentiation.

341 A. Increase in BIM expression upon Dicer deletion in EpiSCs (day 6 post-deletion) and ESCs

342 (day 7 post-deletion). Representative western blot and western blot quantification relative to

$343 \alpha$-TUBULIN (TUB.). B. Change in $\%$ cells with mitochondrial outer membrane

344 permeabilization (MOMP) upon Dicer deletion in EpiSCs (day 5 post-deletion) and ESCs

345 (day 6 post-deletion) relative to un-deleted cells as measured by DiOC6 staining. C. Cleaved-

346 CASPASE 3 (c-CASP3) immunostaining in ESCs and EpiSCs upon Dicer deletion at day 5

347 and 6 respectively. D. Change in \% Annexin V positive cells after $16 \mathrm{~h}$ treatment with $1 \mu \mathrm{M}$

348 sodium arsenite relative to untreated cells. E. Cleaved-CASPASE 3 levels in ESCs and

349 EpiSCs upon treatment for $16 \mathrm{~h}$ with increasing concentrations of thapsigargin.

350 Representative western blot and graph showing average western blot quantification relative to $351 \alpha$-TUBULIN. Average of 3 (A, E) or 4 (B, D) experiments +/- SEM is shown. Students T-

352 Test (A-B and D) or 2-way ANOVA with Šidák correction (E) *p<0.05, **p<0.01, $353 * * * \mathrm{p}<0.001 . \mathrm{ns}=$ not significant.

354 Figure 2. Enhanced activation of the mitochondrial apoptotic pathway in primed 355 pluripotent cells. Apoptosis quantified as \% Annexin V positive ESCs and EpiSCs treated 356 for $24 \mathrm{~h}$ with increasing concentrations of ABT-737. Fold change versus dmso treated cells is 357 shown. B. Cleaved-CASPASE 3 (c-CASP3) levels relative to HEXOKINASE 2 (HK2) in

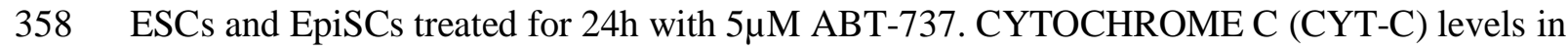
359 the cytosolic and membrane fractions in ESCs and EpiSCs treated with $5 \mu \mathrm{M}$ ABT-737 for 360 24h relative to ERK1/2 and ATP-b. C. Cleaved-CASPASE 3 immunostaining in E3.5 and 361 E6.5 embryos treated with dmso (E3.5 n= 3; E6.5 n=5) or $2 \mu$ M ABT-737 (E3.5 n= 3; E6.5 $362 \mathrm{n}=5$ ) for $1.5 \mathrm{~h}$. D. Fold change in \% Annexin V positive EpiSCs transfected with control 363 siRNA or Bim siRNA and treated with dmso or $5 \mu \mathrm{M}$ ABT-737 for $24 \mathrm{~h}$. E. \% membrane

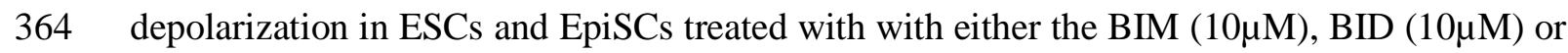
365 control $(10 \mu \mathrm{M})$ peptides for the indicated amounts of time. Average of $3(\mathrm{~A}, \mathrm{E})$ or 4 (B) 366 experiments +/-SEM (D) or +/-SD (E) is shown. (D) Student T-Test or (A) 2-way ANOVA 367 with Šidák correction $* \mathrm{p}<0.05, * * \mathrm{p}<0.01$ or $* * * *<0.00001$.

368 Figure 3. Expression levels of BCL-2 apoptotic factors in pluripotent cells A. Levels of 369 pro-apoptotic factors in whole cell lysate and mitochondrial extract of ESCs an EpiSCs (Epi).

370 Graphs show protein level normalized against $\alpha$-TUBULIN (TUB.) or $\beta$-ACTIN in whole 371 cell lysate and ATP-b in mitochondrial extract. B. Levels of apoptosis effectors in whole cell 372 lysate and mitochondrial extract of ESCs an EpiSCs. Graphs show protein level normalized 373 against $\alpha$ TUBULIN, $\beta$ ACTIN or PCNA for the whole cell lysates and ATP-b for the 374 mitochondrial extracts. C. Levels of anti-apoptotic factors in whole cell lysate and 375 mitochondrial extract of ESCs an EpiSCs. Graphs show protein level normalized against $376 \alpha$ TUBULIN or $\beta$ ACTIN in whole cell lysate and ATP-b in mitochondrial extract. Average of 3774 independent experiments +/-SEM is shown. Students T-Test $* \mathrm{p}<0.05$, **p<0.01, $378 * * * \mathrm{p}<0.001$ 
379 Figure 4. Mitochondria fuse to form complex networks upon differentiation. A. ATP-b

380 immunostaining showing mitochondrial morphology in ESCs and EpiSCs. B. ATP-b 381 immunostaining showing mitochondrial morphology in E3.5, E4.5 and E6.5 mouse embryos.

382 2x magnification over ICM/Epiblast area. C. Basal levels of mitochondrial fusion and fission 383 proteins in ESC and EpiSCs. D. Mitochondrial fusion and fission protein levels in EpiSCs 384 versus ESCs. Protein levels are normalized against $\alpha$-TUBULIN (TUB.). Graph shows the 385 average from 3 independent experiments +/-SEM is shown. 2-way ANOVA with Šidák 386 correction $* * \mathrm{p}<0.01, * * * \mathrm{p}<0.001$ or $* * * *<0.00001$.

387 Figure 5. Drp1 deletion facilitates early apoptotic events. A. ATP-b and NANOG 388 immunostaining showing mitochondrial morphology in wild type and $\operatorname{Drpl}^{-1}$ ESCs. B. 389 Quantitative RT-PCR showing gene expression levels of naïve and primed pluripotency 390 markers in wild-type and Drp1 $1^{-/}$ESCs. Gene expression normalized against Gapdh. C. \% of 391 cells with MOMP detected by TMRM staining in wild-type and Drp1 ${ }^{-/}$ESCs untreated or treated with $1 \mu \mathrm{M}$ sodium arsenite for $16 \mathrm{~h}$ or $\mathbf{D} 1 \mu \mathrm{M}$ Thapsigargin for $16 \mathrm{~h}$. Data normalized against wild-type cells. E. \% cells with MOMP in ESCs and EpiSCs treated with with either the BMI $(0.5 \mu \mathrm{M})$, BID $(2.5 \mu \mathrm{M})$ or control $(1 \mu \mathrm{M})$ peptides for the indicated amounts of time. Average of 3 (B, C), 4 (D) or 7 (E) independent experiments +/-SEM (C, D) or +/-SD (E) is shown. 2-way ANOVA with Šidák correction $* \mathrm{p}<0.05, * * \mathrm{p}<0.01$ or $* * * *<0.00001$.

Figure 6. DRP1 over-expression inhibits the apoptotic response during the onset of pluripotent stem cell differentiation. A. ATP-b immunostaining showing mitochondrial morphology in wild-type and $\operatorname{Drpl} 1^{O E}$ cells during differentiation. B. Circularity measurement of mitochondrial particles from ATP-b immunostained images of wild-type and $\mathrm{Drpl}^{O E}$ cells at day 3 of differentiation in N2B27. C. Quantitative RT-PCR showing gene expression levels of naïve and primed pluripotency markers in wild-type and $D r p 1^{O E}$ ESCs and at day 3 of differentiation in N2B27. Gene expression normalized against Gapdh. D. Fold change in basal cleaved-CASPASE 3 levels in wild-type and $\operatorname{Drpl}^{O E}$ cells at day 3 of differentiation in N2B27. E. Fold change in cleaved-CASPASE 3 levels in wild-type and $\operatorname{Drpl}^{O E}$ cells untreated or treated with $1 \mu \mathrm{M}$ Thapsigargin for $5 \mathrm{~h}$. F. Fold change in cleaved-CASPASE 3 levels in wild-type and $D r p 1^{O E}$ cells untreated or treated with $1 \mu \mathrm{M}$ sodium arsenite for $5 \mathrm{~h}$. G. Fold change in cleaved-CASPASE 3 levels in wild type and $\operatorname{Drpl}^{O E}$ cells untreated or treated with $1 \mu \mathrm{M}$ ABT-737 for $5 \mathrm{~h}$ at day 3 of differentiation in N2B27. Protein levels in D. E. F. and G. are normalized against $\alpha$-TUBULIN and graphs show protein expression levels relative to wild-type cells. Average of 3 (C, E, F) or 5 (D) independent experiments +/- SEM is shown. statistical comparisons are made to untreated cells. 
419 Dicer deletion in ESCs. Expression normalized against sno135. Fold change vs un-deleted 420 cells is shown. D. Change in \% of Annexin V positive ESCs at different time points after 421 Dicer deletion relative to un-deleted cells. E. Change in \% of ESCs and EpiSCs with loss of 422 mitochondrial membrane potential (MOMP) after 24h treatment with $5 \mu$ M ABT-737, relative 423 to dmso treated cells. F. Cleaved-CASPASE 3 levels in E3.5 and E6.5 embryos treated with 424 dmso or $2 \mu \mathrm{M}$ ABT-737 for 1.5h. E3.5 dmso ( $n=5)$, ABT (n=5); E6.5 dmso (n=6) ABT (n=6). 425 Average of a minimum of (B,F) 3, (D) 4 or (E) 5 experiments +/- SEM is shown. 2-way 426 ANOVA with a Turkey correction $(B, G) * p<0.05$, **p $<0.01$ or $* * * p<0.001$.

427 Supplementary Figure 2. Expression of BCL-2 family members upon BCL-2/XL 428 inhibition. A. Levels of anti-apoptotic factors and BIM in whole cell extracts and

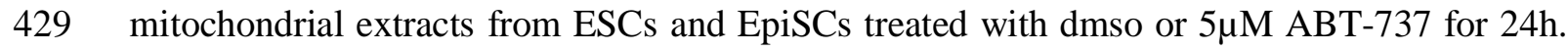
430 A representative western blot is shown. B. Levels of anti-apoptotic proteins and BIM in 431 whole cell and mitochondrial extracts from ESCs and EpiSC (Epi)s treated with dmso or $4325 \mu \mathrm{M}$ ABT-737 for $24 \mathrm{~h}$. Protein level is normalized against $\alpha$-TUBULIN (TUB.) in whole 433 cell extracts and against ATP-b in mitochondrial extracts. Fold change of ABT versus dmso 434 treated is shown. Average of 4 experiments +/- SEM is shown. protein levels in wild type and $\mathrm{Drpl}^{-/}$ESCs. B. Extracellular acidification rate (ECAR) during the glycolysis stress test. C Metabolic parameters assessed during a glycolysis stress test. D. Oxygen consumption rate (OCR) during the mitochondria stress test. E. Metabolic parameters assessed after the mitochondria stress test. F. CYTOCHROME C (CYT-C) protein levels in cytosolic and membrane fractions of wild-type and $\operatorname{Drp}^{-/-}$ESCs un-treated or treated with $1 \mu \mathrm{M}$ Thapsigargin for $6 \mathrm{~h}$. Graph shows cytochrome $\mathrm{C}$ protein levels normalized against ERK1/2 (cytosolic fraction) or ATP-b (membrane fraction). Average of 3 independent experiments +/- SEM is shown. Statistical analysis was done with a (C,E) Mann Whitney test $* \mathrm{p}<0.05, * * \mathrm{p}<0.01$ or $* * * \mathrm{p}<0.001$. Quantification of total DRP1 protein levels in wild-type and $\operatorname{Drpl}^{O E}$ ESCs and differentiating cells. Protein levels are normalized against $\alpha$-TUBULIN (TUB.) and the graph shows expression levels relative to wild-type cells quantified from Figure 5A. B. Levels of phosphoDRP1 (S616) in wild-type and $\operatorname{Drpl}^{O E}$ cells at day 3 of differentiation in N2B27 detected by Western blot. C. Quantification of (B). Protein levels are normalized against $\alpha$ TUBULIN and the graph shows protein expression levels relative to wild-type cells. Average of 3 (C) or 5 (A) independent experiments $+/-$ SEM is shown. Statistical comparisons are made to the control cells in the same culture condition. Students T-Test **p<0.01, ***p $<0,001$. cleaved-CASPASE 3 (c-CASP3), $\alpha$-TUBULIN (TUB.) and NANOG protein levels in wildtype and $\operatorname{Drpl}^{O E}$ ESCs at day 3 of differentiation in N2B27 untreated or treated with $1 \mu \mathrm{M}$ 
458 differentiated cells. Protein levels are normalized against $\alpha$-TUBULIN and graph shows 459 differentiation expression levels relative to ESCs for each cell type. C. Total DRP1, cleaved460 caspase 3 and $\alpha$-TUBULIN protein levels in wild-type and $\operatorname{Drpl}^{O E}$ at day 3 of differentiation 461 in N2B27 untreated or treated with $1 \mu \mathrm{M}$ NaAs for 5h. D. Total DRP1, cleaved-caspase 3 and $462 \alpha$-TUBULIN protein levels in wild-type and $\operatorname{Drpl}^{O E}$ at day 3 of differentiation in N2B27 463 untreated or treated with $1 \mu \mathrm{M}$ ABT-737 for $4 \mathrm{~h}$. The statistical comparisons are made to 464 untreated cells. In B average of 3 independent experiments +/- SEM is shown. The statistical 465 analysis compares a cell line in ESC and differentiation culture conditions . Students T-Test $466 * * * * \mathrm{p}<0.0001$ 


\section{Materials and Methods}

469 Stem Cell culture and treatments:

470 All cells were cultured at $37^{\circ} \mathrm{C}$ in an atmosphere with $5 \% \mathrm{CO}_{2}$. Reagents used for tissue culture were obtained from Invitrogen unless otherwise stated. Mouse embryonic stem cells (ESCs) were cultured on $0.1 \%$ gelatin-coated flasks (Nunc, Thermo Fisher) in GMEM containing with $10 \%(\mathrm{v} / \mathrm{v})$ foetal calf serum (FCS; Seralab), 1X non-essential amino acids, 2 mM L-glutamine, $0.1 \mathrm{mM} \beta$-mercaptoethanol and supplemented with homemade leukaemia inhibitory factor (1:500, LIF). ESCs were routinely dissociated with trypsin and cryopreserved in $10 \%$ DMSO in FCS.

477 Epiblast Stem Cells (EpiSCs) were cultured on FCS coated dishes in N2B27 medium (100mL 478 DMEM F12, 100mL Neurobasal, 1mL N2, 2mL B27 without retinoic acid, 2mM L-

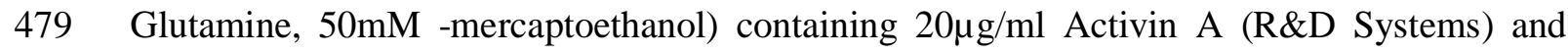
$48012 \mathrm{ng} / \mathrm{ml}$ bFGF (R\&D Systems). Cells were passaged by mechanical disruption as previously 481 described $^{44}$. E14 EpiSC were derived from E14 mESCs as previously described ${ }^{45}$. All 482 experiments were performed using cells in passage between 20 and 30 .

483 To induce ESCs differentiation cells were seeded onto plates coated with fibronectin (Merk) 484 and cultured in N2B27 media (Neurobasal media; DMEM F12 media, 0.5 x B27 supplement; 4850.5 x N2 supplement; $0.1 \mathrm{mM}$ 2-mercaptoetanol; 2mM glutamine; all Thermo Fisher 486 Scientific) during 3 days to allow differentiation.

487 To induce Dicer deletion Dicer ${ }^{f x / f x}$ ESCs and EpiSCs ${ }^{11,46}$ were cultured in the presence of 0.3 $488 \mathrm{mM}$ 4-OH-Tamoxifen for three days and left untreated from the third day onwards as 489 previously described ${ }^{11}$.

490 For BCL2/BCL-XL inhibition ABT-737 (Selleckchem) was added to the media for $24 \mathrm{~h}$ at 491 the stated concentration. Oxidative stress and ER stress were induced by adding sodium 492 arsenite (Sigma Aldrich) or Thapsigargin (Sigma Aldrich) for $16 \mathrm{~h}$ at the stated 493 concentrations. To induce Bim knockdown, a previously tested Bim siRNA (Mm_Bcl2111_2 494 FlexiTube siRNA, Qiagen) ${ }^{11}$ was transfected into EpiSCs at a final concentration of 75nM 495 using HiPerFect transfection reagent (Qiagen) according to manufacturers' instructions. 496 Transfection of Flexi-Tube Negative Control siRNA (Qiagen) at a final concentration of $49775 \mathrm{nM}$ was used as negative control.

\section{Flow cytometry analysis: Annexin V staining and MMP measurement}

499 For apoptosis detection by flow cytometry, Annexin V-APC (Thermo Fisher Scientific) was 500 used in combination with Propidium Iodide (Sigma) according to manufacturer's instructions. 501 Briefly, approximately $2 \times 10^{5}$ cells were stained in $100 \mu$ l of Annexin V Binding Buffer $(0.1 \%$ 502 BSA in $10 \mathrm{mM}$ HEPES, $140 \mathrm{mM} \mathrm{NaCl}, 2.5 \mathrm{mM} \mathrm{CaCl}$, pH7.4) containing APC conjugated 503 Annexin $\mathrm{V}$ for 15 minutes in the dark, after which $0.1 \mathrm{mg} / \mathrm{ml}$ propidium iodide was added 504 and the samples immediately analysed by flow cytometry. Loss of mitochondrial membrane 505 potential was measured using the fluorescent dyes DiOC6 or TMRM. Briefly, 2x10 5 cells 506 were re-suspended in PBS containing 40 nM DiOC6 (Sigma) or 100nM TMRM (T668, 
507 ThermoFisher Scientific), incubated for $15 \mathrm{~min}$ at $37^{\circ} \mathrm{C}$ and analyzed by flow cytometry.

508 Data was acquired with a BD LSRII cytometer and analyzed with the FlowJo software (BD).

509 Stem cells immunofluorescence

510 For immunostaining ESCs and EpiSCs were fixed for 10min in 4\%PFA at room temperature,

511 permeabilised in $0,4 \%$ Triton-X100/PBS for 5 minutes at room temperature, blocked in

$51210 \% \mathrm{BSA} / 0,1 \%$ Triton $\mathrm{X}-100 / \mathrm{PBS}$ and incubated overnight at $4{ }^{\circ} \mathrm{C}$ in primary antibody

513 diluted in 1\%BSA/0,1\% Triton X-100 (anti-Cleaved Caspase 3 Asp175, Cell Signalling,

514 1/100; anti-Nanog (14-5761 $\square 80$ eBioscience, 1/100, anti ATP-b (Ab14730, Abcam - 1:200).

515 Alexa-Fluor conjugated secondary antibodies (Thermo Fisher Scientific) were used at 1/500

516 dilution in 1\%BSA/0,1\% Triton X-100. Cells were mounted for visualization in Vectashield

517 with DAPI (Vector Laboratories). Images were acquired with a Zeiss confocal microscope

518 and analysed with the Fiji software ${ }^{47}$.

519 Mitochondrial Staining

520 Cells were washed with PBS and fixed with 3.7\% formaldehyde diluted in serum free media

521 for $15 \mathrm{~min}$ at $37^{\circ} \mathrm{C}, 5 \% \mathrm{CO}_{2}$. Cell were washed two times with PBS and incubated with pre-

522 cooled acetone for $5 \mathrm{~min}$ at $-20^{\circ} \mathrm{C}$. Cells were washed two times with PBS and incubated

523 with blocking/permeabilization (5\% BSA, 0.4\% Triton-X in PBS) solution for 30 min at RT

524 before incubating with the primary antibody $\mathrm{O} / \mathrm{N}$ at $4{ }^{\circ} \mathrm{C}$. Excess antibody was removed and

525 cells washed three times in PBS, then incubated with the secondary antibody for $45 \mathrm{~min}$ at

526 RT. Before mounting with Vectashield with DAPI, secondary antibody was removed and cell

527 washed again three times in PBS.

528 Images were acquired in a LSM Z800 Confocal microscope and processed with FIJI. Images

529 for deconvolution were acquired with the same microscope and further processed with the

530 software Huygens. Deconvolution analysis was performed with the support and advice from

531 Mr. Stephen Rothery from the Facility for Imaging by Light Microscopy (FILM) at Imperial

532 College London.

533 Primary antibodies used for immunofluorescence: Tom20 (1/100, Santa Cruz), ATP-b (1:100,

534 Abcam), Nanog (1:100, eBioscience), Gata4 (1:100, Santa Cruz), Sox2 (1:100, R\&D). Alexa-

535 Fluor conjugated secondary antibodies (Invitrogen) were used at a concentration of 1:600.

536 Mitochondria circularity measurements were done with a plugin from FIJI that calculates

537 object circularity according to the formula circularity $=4 \pi\left(\right.$ area/perimeter $\left.{ }^{2}\right)$. A circularity

538 value of 1.0 indicates a perfect circle. As the value approaches 0.0 , it indicates an

539 increasingly elongated polygon. The calculations were done on ATP-b immunostained

540 images.

\section{Western blot analysis}

542 Western blot was performed according to established protocols described elsewhere ${ }^{11}$.

543 Briefly, protein samples were collected in Laemmli buffer and denatured for 10 minutes at

$54495^{\circ} \mathrm{C}$. All samples were run in polyacrylamide gels and transferred to nitrocellulose

545 membranes. Blocking was performed in 5\% milk in TBST buffer and primary antibody 
546 incubation was done overnight at $4^{\circ} \mathrm{C}$ in TBST containing 5\% BSA. The following antibodies 547 were used at the stated concentration: anti-Bim/Bod (Enzo, 1/1000), anti-Puma (Abcam, 548 1/1000), anti- Bid (Cell Signaling, 1/1000), anti- Bad (Santa Cruz Biotechnology, 1/500), 549 anti-Bax N20 (Santa Cruz Biotechnology, 1/1000), anti-Bcl2 (BioLegend, 1/500), anti-Bcl550 XL (Santa Cruz Biotechnology, 1/1000), anti- MCL1 (Rockland, 1/10000), anti-Bcl2A1 551 (R\&D Systems, 1/500), anti-Hexokinase II (Cell Signaling, 1/1000), anti-ATP-b (Abcam, 552 1/1000), anti-c C (BD Pharmigen, 1/1000), anti-Erk1/2 (Sigma, 1/20000), anti-Cleaved 553 Caspase 3 Asp175 (Cell Signaling, 1/1000), anti-alpha Tubulin (Cell Signaling, 1/2000), anti554 beta Actin (Santa Cruz Biotechnology, 1/1000), anti-Bak (Millipore, 1/1000) anti-Oct3/4 555 (Santa Cruz Biotechnology, 1/1000), anti-Nanog (eBiosciences, 1/1000), anti-Tom20 (Santa 556 Cruz Biotechnologies, 1/1000), anti-Drp1 (Cell Signalling, 1/1000) anti- pDRP1 (S616) (Cell 557 Signaling 1/1000), anti-Mfn1 (Abcam, 1/1000), anti-Mfn2 (Abcam, 1/1000), anti-Opa1 (BD, 558 1/1000). Western blot quantification was performed using the Fiji software ${ }^{47}$.

559 Mitochondria purification and cytochrome C release assay

560 For mitochondria purification cells were trypsinized and washed twice in 10 packed cell 561 volumes of $1 \mathrm{mM}$ TrisHCl pH 7.4, 0.13M NaCl, $5 \mathrm{mM} \mathrm{KCl}, 7.5 \mathrm{mM} \mathrm{MgCl}_{2}$ followed by 562 centrifugation at $370 \mathrm{~g}$ for 10 minutes. After the second wash pelleted cells were re-suspended 563 in 6 packed cell volumes of homogenization buffer $(10 \mathrm{mM}$ TrisHCl pH6.7, $10 \mathrm{mM} \mathrm{KCl,} 0.15$ $564 \mathrm{mM} \mathrm{MgCl} 2,1 \mathrm{mM}$ PMSF, $1 \mathrm{mM}$ DTT) and incubated for 10 minutes on ice. Cells were 565 homogenized in a glass homogenizer until achieving approximately $60 \%$ cell breakage. 566 Homogenate was poured into a tube containing 1 packed cell volume of $2 \mathrm{M}$ sucrose solution, 567 mixed and centrifuged at $1200 \mathrm{~g}$ for 5 minutes to pellet unbroken cells, nuclei and debris. This 568 treatment was repeated twice discarding the pellet, followed by 10 minutes centrifugation at $7000 \mathrm{~g}$ in order to pellet the mitochondria. Mitochondrial pellet was re-suspended in 3 packed cell volumes of mitochondrial suspension buffer (10mM TrisHCl pH6.7, $0.15 \mathrm{mM} \mathrm{MgCl}$, $0.25 \%$ sucrose, $1 \mathrm{mM}$ PMSF, $1 \mathrm{mM}$ DTT) and centrifuged for 5 minutes at $9500 \mathrm{~g}$. Final mitochondrial pellet was re-suspended in 1x Laemmli Buffer and boiled at $95^{\circ} \mathrm{C}$ for 10 573 minutes for western blot analysis.

574 For the separation of cytosolic and membrane fractions in order to investigate cytochrome C 575 release, cells were washed twice with ice cold PBS and incubated for 10 minutes rocking on 576 ice in Digitonin Buffer (20mM HEPES/KOH pH7.5, $100 \mathrm{mM}$ sucrose, 2.5mM $\mathrm{MgCl}_{2}$, 577 100mM KCL, 1mM DTT, $0.0025 \%$ digitonin) supplemented with Complete protease 578 inhibitors (Roche). The supernatant was collected as cytosolic fraction and Triton X-100 579 Extraction Buffer $^{48}$ was added to the plates followed by 30 minute incubation rocking on ice. 580 The resulting supernatant was taken as membrane fraction.

\section{Embryo culture, treatment and immunofluorescence}

582 E3.5 embryos were obtained by flushing of the uterus at 3.5 days post coitum and cultured in 583 M16 media containing DMSO, $1 \mu$ M Etoposide (Sigma) or $2 \mu \mathrm{M}$ ABT-737 (Selleckchem). 584 E6.5 embryos were dissected from pregnant females at 6.5 days post coitum and cultured in 
585 N2B27 media (see Epiblast Stem Cell culture conditions) containing DMSO, $5861 \mu \mathrm{M}$ Etoposide or $2 \mu \mathrm{M}$ ABT-737.

587 For immunostaining, embryos were fixed in $4 \%$ PFA/0.1\% Tween/0.01\% Triton X588 100/PBS (10 $\mathrm{min}$ for $3.5 \mathrm{dpc}$ and $4.5 \mathrm{dpc}, 20 \mathrm{~min}$ for $6.5 \mathrm{dpc}$ ) at room temperature, 589 permeabilized in $0.5 \%$ Triton X-100/PBS for $15 \mathrm{~min}(3.5 \mathrm{dpc}$ and $4.5 \mathrm{dpc})$ or $20 \mathrm{~min}(6.5 \mathrm{dpc})$, 590 washed in $0.1 \%$ Triton X-100/PBS and blocked in $2 \%$ Horse serum in $0.1 \%$ Triton X591 100/PBS for 45 minutes at room temperature. Primary antibodies (anti-Cleaved Caspase 3 592 Asp175, Cell Signaling, 1/100; anti-p53 (1C12), Cell Signalling, 1/100, anti ATP-b, 593 (Ab14730, Abcam - 1:200) were incubated overnight at $4^{\circ} \mathrm{C}$ in $0.2 \%$ Horse serum in $0.01 \%$ 594 Triton X-100/PBS. Embryos were incubated with Alexa-Fluor conjugated secondary 595 antibodies (Thermo Fisher Scientific) for $1 \mathrm{~h}$ at $4^{\circ} \mathrm{C}$ and counterstained with $6 \mathrm{ug} / \mathrm{ml} \mathrm{Hoechst}$ 596 for nuclear visualization. Images were acquired with a Zeiss confocal microscope and 597 analyzed with the Fiji softwar ${ }^{47}$.

\section{Embryo image quantification}

599 Corrected cell fluorescence was measured using (Fiji, Image J) as previously described 600 (reference below). An outline was drawn around each embryo. Area, mean fluorescence and 601 integrated density were measured. In addition, adjacent areas were also selected an measured 602 as background readings. Corrected cellular fluorescence (CCF) was calculated using the 603 formula: $\mathrm{CCF}=$ integrated density - (area of selected cell $\times$ mean fluorescence of 604 background readings. Cleaved Caspase 3 signal was normalised to DAPI ${ }^{49}$.

\section{BH3 profiling}

606 The assay was performed following the protocol described by Anthony Letai's laboratory ${ }^{27}$.

607 Briefly, $15 \mu \mathrm{L}$ of $2 \mathrm{x}$ peptides diluted in DTEB buffer (135mM threalose, 10mM HEPES KOH

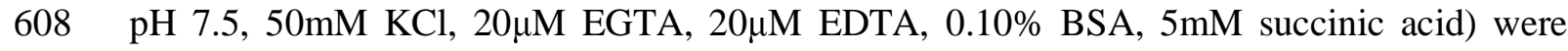
609 added to each well of a dark 384 well plate (Nunc). Cells were dissociated with trypsin, 610 counted and resuspended at $2.67 \times 10^{6}$ cells $/ \mathrm{mL}$. Equal volume of cells and dying mix (4x 611 digitonin $(0.01 \%), 4 \mathrm{x} \mathrm{JC}-1(4 \mathrm{mM})$ and Oligomycin $(40 \mu \mathrm{g} / \mathrm{mL})$ ) were incubated for 10 612 minutes at room temperature protected from light. $15 \mu \mathrm{L}$ of this mix was added per well of the 613384 well plate. DMSO and CCCP (Sigma) were used as no-depolarization and full 614 depolarization controls. BID, BIM, BMF and a peptide control were tested in this profile. 615 Each sample was loaded by triplicate and at least three biological replicates were analysed. 616 Fluorescence was measured every 5 minutes for a period of 70 minutes, with a 544/590 filter 617 in a FluoStar Omega plate reader (BMG Omega). Percentage of depolarization was 618 calculated by normalizing the data to the membrane potential of cells that have not been 619 exposed to peptides but have been treated with DMSO (vehicle) or CCCP, a protonophore 620 that causes complete mitochondrial depolarization ${ }^{27}$.

621

$$
\% \text { Depolarization }=\left(1-\frac{(\text { Peptide }-\mathrm{CCCP})}{(\mathrm{DMSO}-\mathrm{CCCP})}\right) \cdot 100
$$


PEPTIDE

BIM

BID

BFM

CONTROL
SEQUENCE

Ac-MRPEIWIAQELRRIGDEFNA-

$\mathrm{NH} 2$

Ac-EDIIRNIARHLAQVGDSMDRY-

$\mathrm{NH} 2$

Ac-HQAEVQIARKLQLIADQFHRY-

$\mathrm{NH} 2$

Ac-EQWAREIGAQARRMAADLNA-

$\mathrm{NH} 2$
624

625

626

627

628

629

630

631

632

633

634

635

636

637

638

639

640

641

642

643

644

645

646

647

648

649

650

651

652

653

654

\section{Generation of Drp1 Knock-Out and Drp1 Overexpressing cells}

Drpl knockout ESCs were generated by CRISPR-Cas9 mediated deletion of Drpl exon 2. sgRNA guides flanking Drpl exon2 were cloned into the PX459 vector (Addgene) ${ }^{50}$ : Drpl exon 2 upstream sgRNA: 5' TGGAACGGTCACAGCTGCAC 3'; Drpl exon 2 downstream sgRNA: 5' TGGTCGCTGAGTTTGAGGCC 3'. E14 ESCs were co-transfected with 1 ug of each sgRNA expression using Lipofectamine 2000 (Invitrogen) according to manufacturer's instructions. As control E14 ESCs were transfected in parallel with equal amount of empty PX459 plasmid. Following 6 days of Puromycin selection, single colonies were picked from both Drpl sgRNA and empty vector transfected ESCs and screened for mutations. Drpl exon 2 deletion was confirmed by PCR genotyping using the following primers: Drpl_genot F: 5' GGATACCCCAAGATTTCTGGA 3'; Drp1_genot R: 5' AGTCAGGTAATCGGGAGGAAA 3', followed by Sanger Sequencing.

Drp1 overexpressing cells were generated by transfecting ESCs with a pCAG-Drp1 plasmid. To generate this plasmid the Drpl cDNA (Addgene plasmid 45160) was cloned into a pCAG mammalian expression plasmid. E14 ESCs were transfected with 2 ug of the pCAG-Drp1 plasmid using Lipofectamine 2000 (Invitrogen) according to manufacturer's instructions. Following 6 days of Puromycin selection, single colonies were picked and analysed for DRP1 expression by western blot.

\section{RNA Extraction and Quantitative RT-PCR}

RNA was extracted with the RNeasy mini kit (Qiagen) and SuperScript III reverse transcriptase (Thermo Fisher Scientific) was used for cDNA synthesis according to manufacturer's instructions. Quantitative RT-PCR was performed by amplification with Lightcycler 480 SYBR green Master (Roche). The primers used are listed in Table 1. RNA samples from wild type and mutant clones were collected from 3 independent experiments.

For miRNA q-PCR total RNA was extracted using the mirVana miRNA isolation kit (Ambion) and cDNA was synthesized with the TaqMan miRNA reverse transcription kit (Applied Biosystems). qPCR for individual miRNAs was performed using TaqMan probes and TaqMan universal PCR master mix (Applied Biosystems). miRNA expression was normalized against sno135. 
Table 1: Primers used in quantitative RT-PCR

656

657

658

659

660

661

662

663

664

665

666

667

668

669

670

671

672

673

674

675

676

677

678

679

680

681

682

683

\begin{tabular}{|ccc|}
\hline Gene & Forward & Reverse \\
\hline Gapdh & 5'CATGGCCTTCCGTGTTCCTA 3' & 5' GCGGCACGTCAGATCCA 3' \\
\hline $\boldsymbol{F g} \boldsymbol{f 5}$ & 5' AAAGTCAATGGCTCCCACGAA 3' & 5' CTTCAGTCTGTACTTCACTGG 3' \\
\hline $\boldsymbol{E s \boldsymbol { r } \boldsymbol { b }}$ & 5' GGACACACTGCTTTGAAGCA 3' & 5' ACAGATGTCTCTCATCTGGC 3' \\
\hline $\boldsymbol{N a n o g}$ & 5'CTTACAAGGGTCTGCTACTGAGATGC 3' & 5' TGCTTCCTGGCAAGGACCTT 3' \\
\hline $\boldsymbol{R e x \boldsymbol { l }}$ & 5' CGAGTGGCAGTTTCTTCTTGG 3' & 5' GACTCACTTCCAGGGGGCAC 3' \\
\hline
\end{tabular}

\section{Seahorse Analysis}

The metabolic function of cells was assessed by extracellular flux analysis using Seahorse XF24 (Agilent Technologies, UK). On the day prior to the assay, $5 \times 10^{4}$ cells per well were seeded on $0.1 \%$ gelatin-coated (Sigma, UK) 24-well plates and grown in $300 \mu \mathrm{L}$ of pluripotency maintenance conditions. Cells were washed, just before the assay, with the assay media and left with a final volume of the $600 \mu \mathrm{L}$ per well. The plate was then equilibrated on a non- $\mathrm{CO}_{2}$ incubator at $37^{\circ} \mathrm{C}$ for $30 \mathrm{~min}$. The assay media consisted in unbuffered DMEM (D5030 - Sigma, UK), reconstituted with 1.83 g.L $\mathrm{L}^{-1}$ of $\mathrm{NaCl}$ in $\mathrm{dH}_{2} \mathrm{O}$, that was supplemented on the day of the assay according to the test performed. For the OCR measurements the assay media was supplemented with 0.5 g. $\mathrm{L}^{-1}$ of glucose (Sigma, UK) and $2 \mathrm{mM}$ of L-glutamine (Life Technologies, UK), while for the ECAR measurements the media was supplemented with $1 \mathrm{mM}$ of Sodium Pyruvate and $2 \mathrm{mM}$ of L- glutamine (both from Life Technologies, $\mathrm{UK}), \mathrm{pH} 7.4$ at $37^{\circ} \mathrm{C}$.

Assays were performed with 5 biological replicates of each cell type and 4 wells were left without cells for background correction measurements. Both ECAR and OCR measurements were performed on the same plate. The assay parameters for both tests were calculated following the Seahorse assay report generator (Agilent Technologies, UK).

The protocol for the assay consisted of 4 baseline measurements and 3 measurements after each compound addition. Compounds (all from Sigma, UK) used in OCR and ECAR tests were prepared in the supplemented assay media. For the OCR, test the following compounds were added: $2.5 \mu \mathrm{M}$ oligomycin (OM), $300 \mathrm{nM}$ Carbonyl cyanide-4-(trifluoromethoxy) phenylhydrazone (FCCP) and a mixture of rotenone and antimycin A at $6 \mu \mathrm{M}$ each (R\&A). For the ECAR test, the following compounds were added: $2.5 \mathrm{mM}$ and $10 \mathrm{mM}$ of glucose, $2.5 \mu \mathrm{M}$ of oligomycin (OM), and a $50 \mathrm{mM}$ of 2-deoxyglucose (2-DG).

At the end of the assay, cells were fixed and stained with Hoechst. Both OCR and ECAR were normalised to cell number, determined by manual cell counts using FIJI software. 


\section{Statistical methods}

685 Statistical analysis was performed using GraphPad Prism software. Statistical methods used 686 are indicated in the relevant figure legends. No randomization or blinding was used in 687 experiments. Sample sizes were selected based on the observed effects and listed in the figure 688 legends.

689 


\section{References}

691 1. Bowling, S., Lawlor, K. \& Rodriguez, T.A. Cell competition: the winners and losers of fitness selection. Development 146 (2019).

2. Snow, M.H.L. Gastrulation in the mouse: growth and regionalization of the epiblast. J. Embryol. exp. Morph. 42, 293-303 (1977).

3. Leitch, H.G., Tang, W.W. \& Surani, M.A. Primordial germ-cell development and

epigenetic reprogramming in mammals. Curr Top Dev Biol 104, 149-187 (2013).

4. Manova, K. et al. Apoptosis in mouse embryos: elevated levels in pregastrulae and in the distal anterior region of gastrulae of normal and mutant mice. Dev Dyn 213, 293308 (1998).

5. Spruce, T. et al. An early developmental role for miRNAs in the maintenance of extraembryonic stem cells in the mouse embryo. Dev Cell 19, 207-219 (2010).

6. Heyer, B.S., MacAuley, A., Behrendtsen, O. \& Werb, Z. Hypersensitivity to DNA damage leads to increased apoptosis during early mouse development. Genes Dev 14, 2072-2084 (2000).

7. Brown, E.J. \& Baltimore, D. ATR disruption leads to chromosomal fragmentation and early embryonic lethality. Genes Dev 14, 397-402 (2000).

8. Dobles, M., Liberal, V., Scott, M.L., Benezra, R. \& Sorger, P.K. Chromosome missegregation and apoptosis in mice lacking the mitotic checkpoint protein Mad2. Cell 101, 635-645 (2000).

9. Kalitsis, P., Earle, E., Fowler, K.J. \& Choo, K.H. Bub3 gene disruption in mice reveals essential mitotic spindle checkpoint function during early embryogenesis. Genes Dev 14, 2277-2282 (2000).

10. Bolton, H. et al. Mouse model of chromosome mosaicism reveals lineage-specific depletion of aneuploid cells and normal developmental potential. Nat Commun 7, 11165 (2016).

11. Pernaute, B. et al. MicroRNAs control the apoptotic threshold in primed pluripotent stem cells through regulation of BIM. Genes Dev 28, 1873-1878 (2014).

12. Tait, S.W. \& Green, D.R. Mitochondrial regulation of cell death. Cold Spring Harb Perspect Biol 5 (2013).

13. Prudent, J. \& McBride, H.M. The mitochondria-endoplasmic reticulum contact sites: a signalling platform for cell death. Curr Opin Cell Biol 47, 52-63 (2017).

14. Chen, H. et al. Mitofusins Mfn1 and Mfn2 coordinately regulate mitochondrial fusion and are essential for embryonic development. J Cell Biol 160, 189-200 (2003).

15. Smirnova, E., Griparic, L., Shurland, D.L. \& van der Bliek, A.M. Dynamin-related protein Drp1 is required for mitochondrial division in mammalian cells. Mol Biol Cell 12, 2245-2256 (2001).

16. Frezza, C. et al. OPA1 controls apoptotic cristae remodeling independently from mitochondrial fusion. Cell 126, 177-189 (2006).

17. Otera, H., Miyata, N., Kuge, O. \& Mihara, K. Drp1-dependent mitochondrial fission via MiD49/51 is essential for apoptotic cristae remodeling. J Cell Biol 212, 531-544 (2016).

18. Estaquier, J. \& Arnoult, D. Inhibiting Drp1-mediated mitochondrial fission selectively prevents the release of cytochrome c during apoptosis. Cell Death Differ 14, 10861094 (2007). 
735 19. Frank, S. et al. The role of dynamin-related protein 1, a mediator of mitochondrial

736

737

738

739

740

741

742

743

744

745

746

747

748

749

750

751

752

753

754

755

756

757

758

759

760

761

762

763

764

765

766

767

768

769

770

771

772

773

774

775

776

777

778

779 fission, in apoptosis. Dev Cell 1, 515-525 (2001).

20. Karbowski, M. et al. Spatial and temporal association of Bax with mitochondrial fission sites, Drp1, and Mfn2 during apoptosis. J Cell Biol 159, 931-938 (2002).

21. Breckenridge, D.G., Stojanovic, M., Marcellus, R.C. \& Shore, G.C. Caspase cleavage product of BAP31 induces mitochondrial fission through endoplasmic reticulum calcium signals, enhancing cytochrome c release to the cytosol. J Cell Biol 160, 11151127 (2003).

22. Montessuit, S. et al. Membrane remodeling induced by the dynamin-related protein Drp1 stimulates Bax oligomerization. Cell 142, 889-901 (2010).

23. Renault, T.T. et al. Mitochondrial shape governs BAX-induced membrane permeabilization and apoptosis. Molecular cell 57, 69-82 (2015).

24. Lima, A., Burgstaller, J., Sanchez-Nieto, J.M. \& Rodriguez, T.A. The Mitochondria and the Regulation of Cell Fitness During Early Mammalian Development. Curr Top Dev Biol 128, 339-363 (2018).

25. Stern, S., Biggers, J.D. \& Anderson, E. Mitochondria and early development of the mouse. J Exp Zool 176, 179-191 (1971).

26. Nichols, J. \& Smith, A. Naive and primed pluripotent states. Cell stem cell 4, 487-492 (2009).

27. Certo, M. et al. Mitochondria primed by death signals determine cellular addiction to antiapoptotic BCL-2 family members. Cancer Cell 9, 351-365 (2006).

28. Korsmeyer, S.J. Bcl-2 initiates a new category of oncogenes: regulators of cell death. Blood 80, 879-886 (1992).

29. Shepard, T.H., Muffley, L.A. \& Smith, L.T. Mitochondrial ultrastructure in embryos after implantation. Hum Reprod 15 Suppl 2, 218-228 (2000).

30. Zhou, W. et al. HIF1alpha induced switch from bivalent to exclusively glycolytic metabolism during ESC-to-EpiSC/hESC transition. EMBO J 31, 2103-2116 (2012).

31. Knott, A.B., Perkins, G., Schwarzenbacher, R. \& Bossy-Wetzel, E. Mitochondrial fragmentation in neurodegeneration. Nat Rev Neurosci 9, 505-518 (2008).

32. Sancho, M. et al. Competitive interactions eliminate unfit embryonic stem cells at the onset of differentiation. Dev Cell 26, 19-30 (2013).

33. Laurent, A. \& Blasi, F. Differential DNA damage signalling and apoptotic threshold correlate with mouse epiblast-specific hypersensitivity to radiation. Development 142, 3675-3685 (2015).

34. Liu, J.C. et al. High mitochondrial priming sensitizes hESCs to DNA-damage-induced apoptosis. Cell stem cell 13, 483-491 (2013).

35. Csordas, G., Weaver, D. \& Hajnoczky, G. Endoplasmic Reticulum-Mitochondrial Contactology: Structure and Signaling Functions. Trends Cell Biol 28, 523-540 (2018).

36. Booth, D.M., Enyedi, B., Geiszt, M., Varnai, P. \& Hajnoczky, G. Redox Nanodomains Are Induced by and Control Calcium Signaling at the ER-Mitochondrial Interface. Molecular cell 63, 240-248 (2016).

37. Ishihara, N. et al. Mitochondrial fission factor Drp1 is essential for embryonic development and synapse formation in mice. Nat Cell Biol 11, 958-966 (2009).

38. Elgass, K., Pakay, J., Ryan, M.T. \& Palmer, C.S. Recent advances into the understanding of mitochondrial fission. Biochim Biophys Acta 1833, 150-161 (2013). 
780 39. Zhong, X. et al. Mitochondrial Dynamics Is Critical for the Full Pluripotency and Embryonic Developmental Potential of Pluripotent Stem Cells. Cell Metab 29, 979992 e974 (2019).

40. Bahat, A. et al. MTCH2-mediated mitochondrial fusion drives exit from naive pluripotency in embryonic stem cells. Nat Commun 9, 5132 (2018).

41. Xie, Q. et al. Mitochondrial control by DRP1 in brain tumor initiating cells. Nat Neurosci 18, 501-510 (2015).

42. Kashatus, J.A. et al. Erk2 phosphorylation of Drp1 promotes mitochondrial fission and MAPK-driven tumor growth. Molecular cell 57, 537-551 (2015).

43. Nagdas, S. et al. Drp1 Promotes KRas-Driven Metabolic Changes to Drive Pancreatic Tumor Growth. Cell Rep 28, 1845-1859 e1845 (2019).

44. Brons, I.G. et al. Derivation of pluripotent epiblast stem cells from mammalian embryos. Nature 448, 191-195 (2007).

45. Guo, G. et al. KIf4 reverts developmentally programmed restriction of ground state pluripotency. Development 136, 1063-1069 (2009).

46. Nesterova, T.B. et al. Dicer regulates Xist promoter methylation in ES cells indirectly through transcriptional control of Dnmt3a. Epigenetics Chromatin 1, 2 (2008).

47. Schindelin, J. et al. Fiji: an open-source platform for biological-image analysis. Nature methods 9, 676-682 (2012).

48. Ramsby, M. \& Makowski, G. Differential detergent fractionation of eukaryotic cells. Cold Spring Harb Protoc 2011, prot5592 (2011).

49. McCloy, R.A. et al. Partial inhibition of Cdk1 in $\mathrm{G} 2$ phase overrides the SAC and decouples mitotic events. Cell Cycle 13, 1400-1412 (2014).

50. Ran, F.A. et al. Genome engineering using the CRISPR-Cas9 system. Nat Protoc 8 , 2281-2308 (2013). 


\section{Figure 1}

A

Dicer deletion

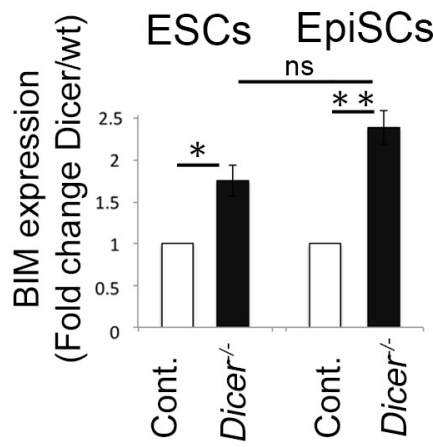

Dicer deletion
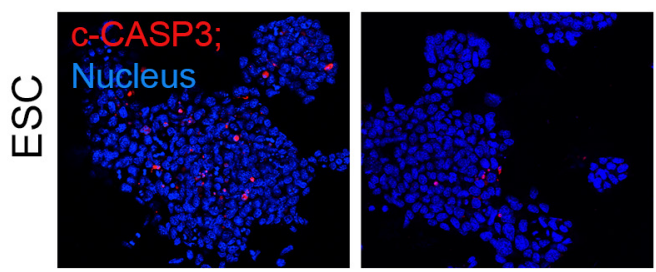

을

ESCs EpiSCs

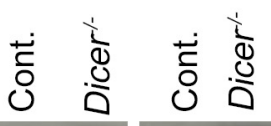

BIM

TUB.
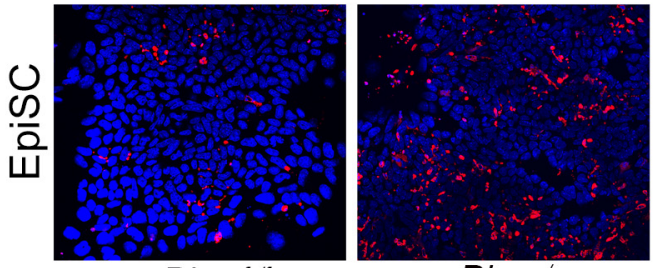

$\operatorname{Dice}^{f x / f x}$

Dicer $^{\prime}$

ER Stress

ESCs

EpiSCs

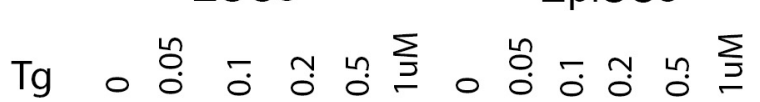

C-CASP3

TUB.
B

Dicer deletion
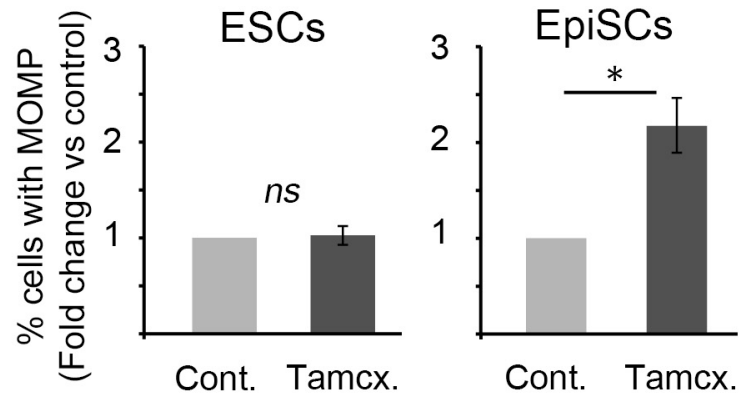

Oxidative stress
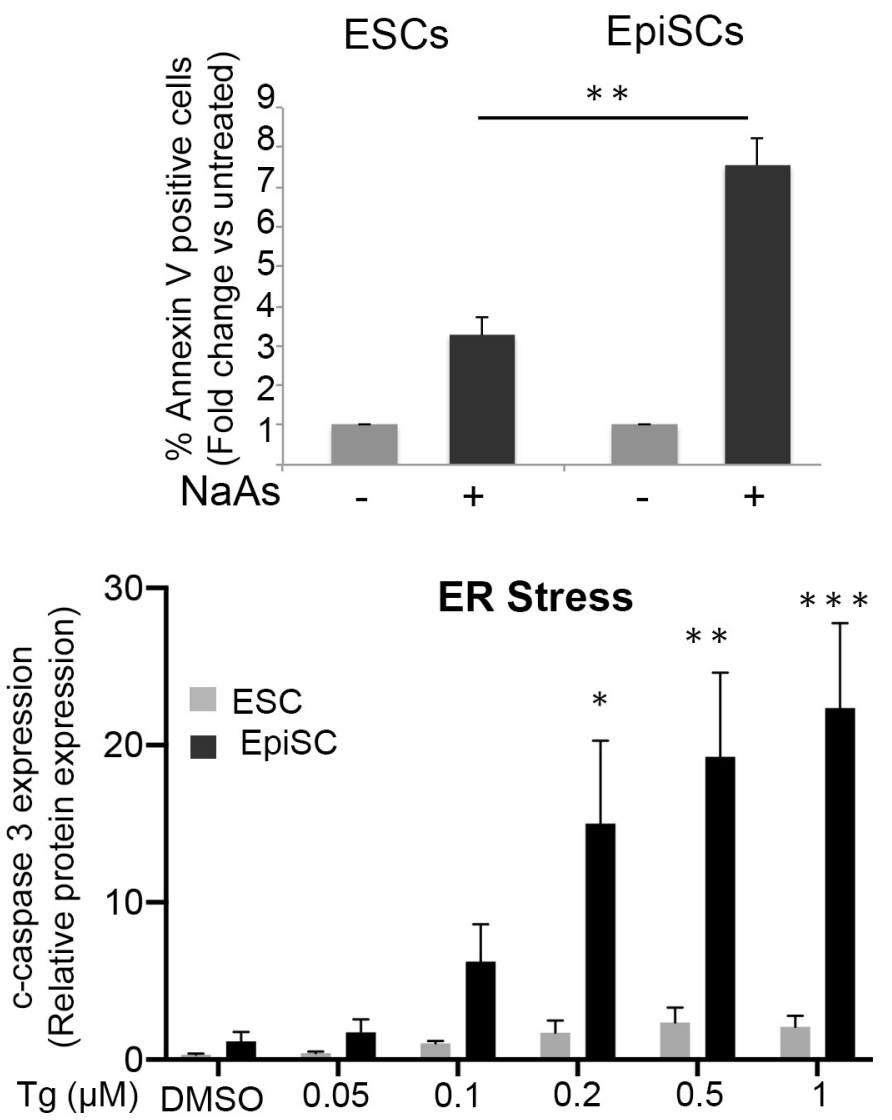
Figure 3

A
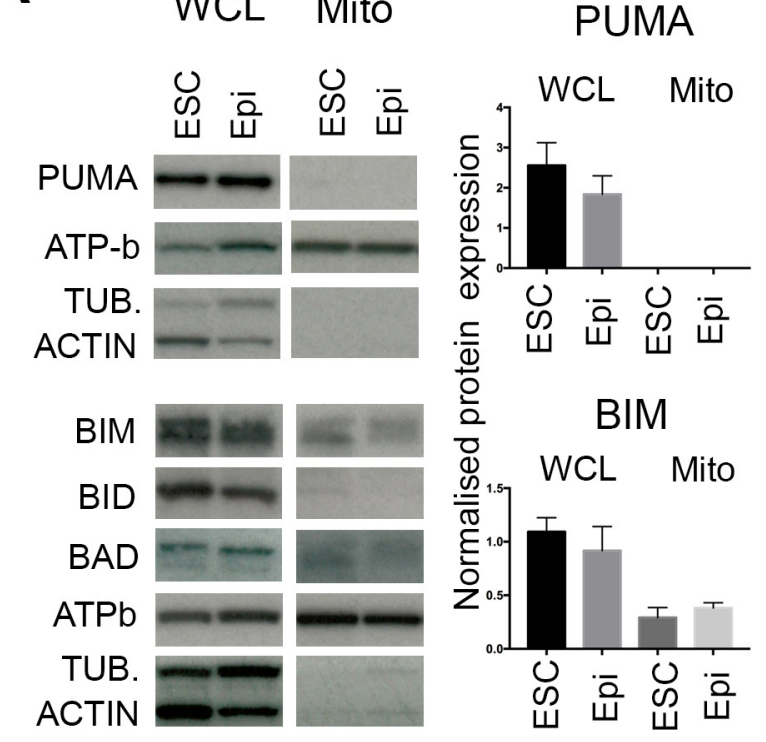

WCL

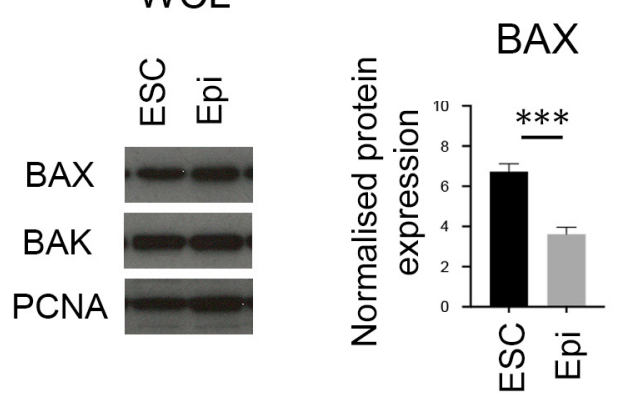

BID
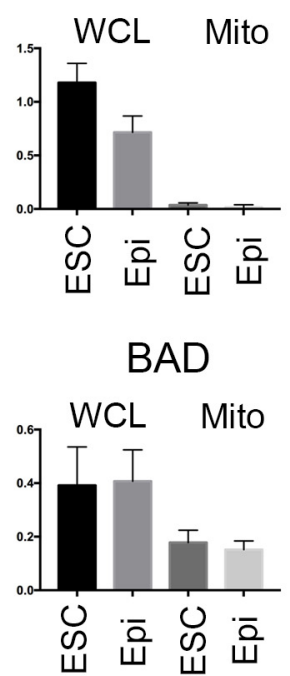

C

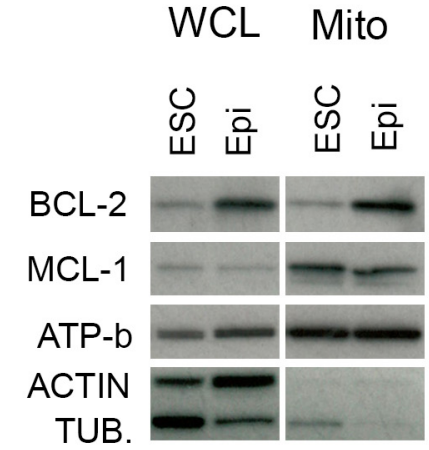

A1

BCL-XL

ATPb

TUB

ACTIN
BCL-2

WCL Mito

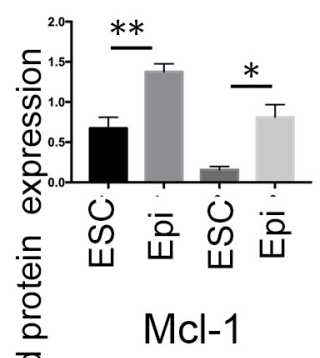

D্ ${ }^{1.5}$ WCL Mito

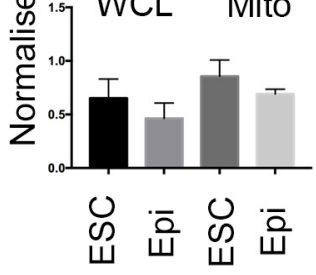

Mito

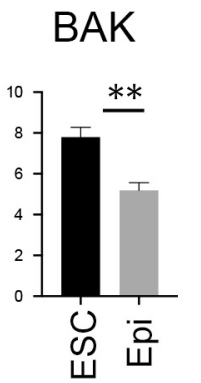

BAX

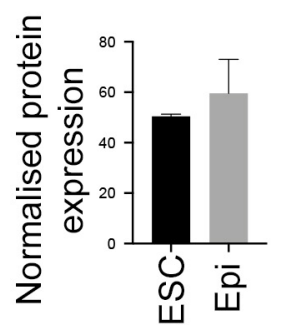

A1

WCL Mito
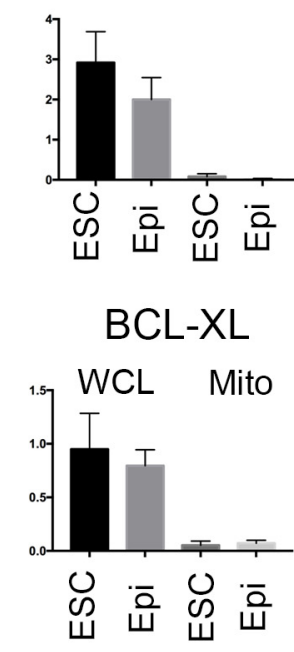

BAK

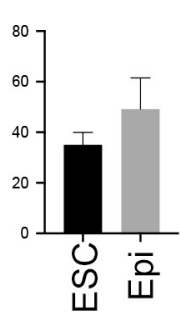




\section{Figure 4}

\section{A}

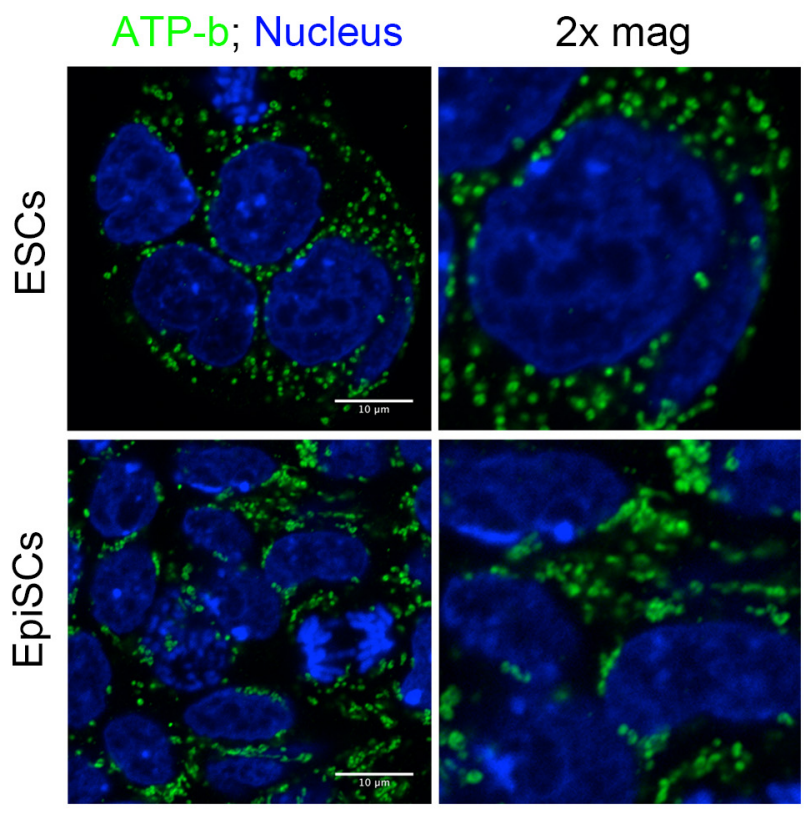

C

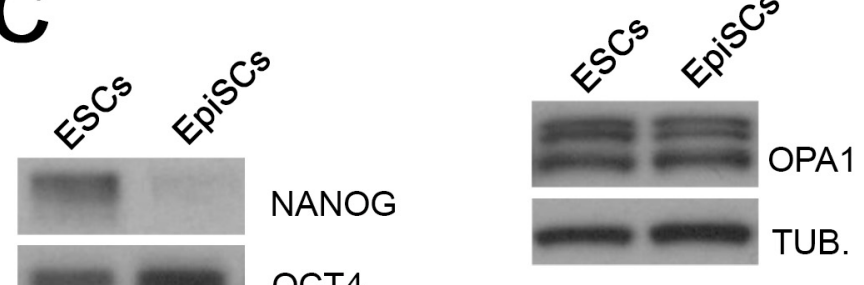

OCT4

BIM

TOM20

DRP1

p-DRP1 (616)

MFN1

MFN2

TUB.

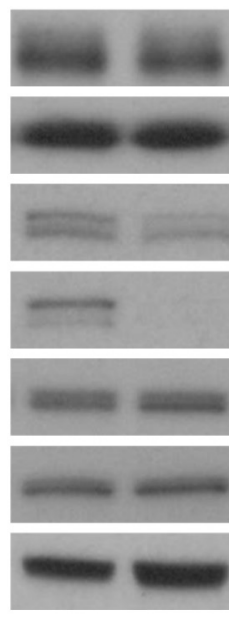

B

ATP-b; Nucleus
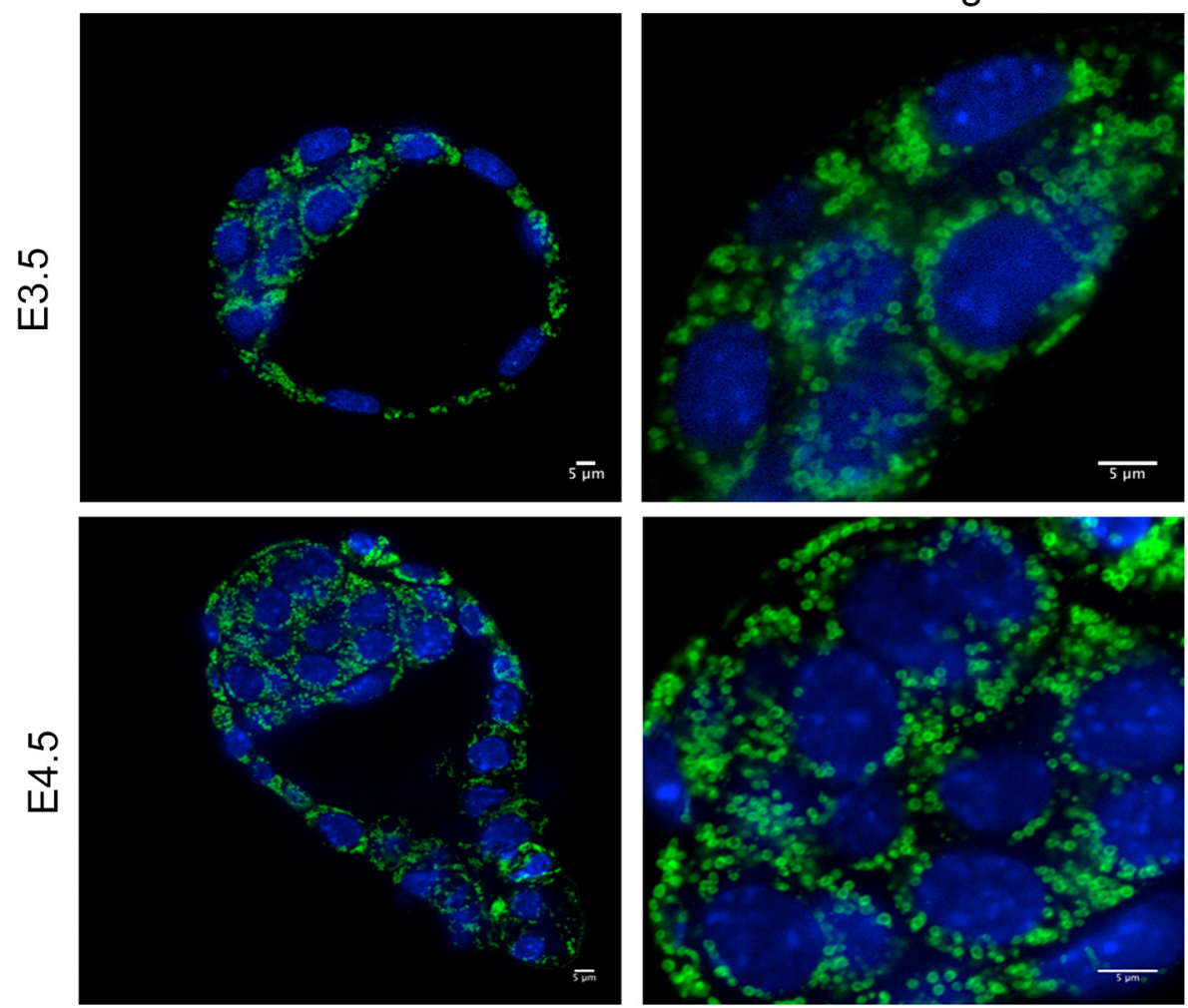

10
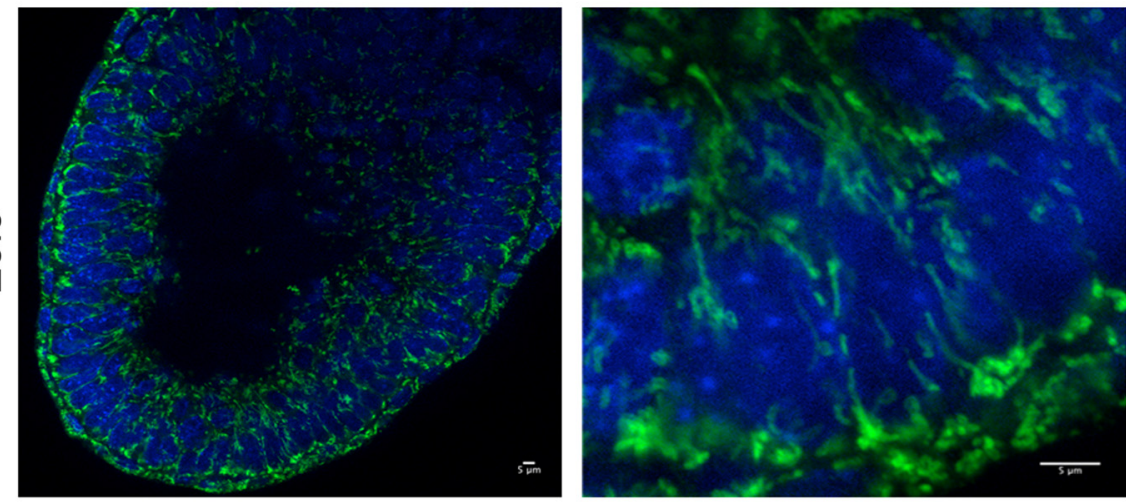

2x mag

3

.

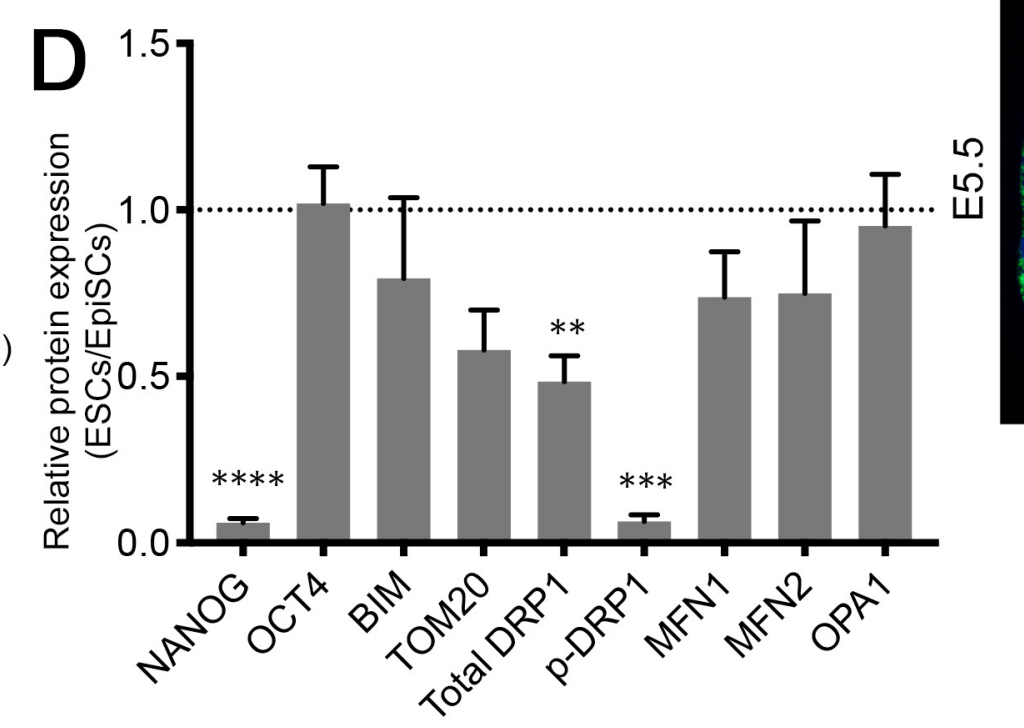


A

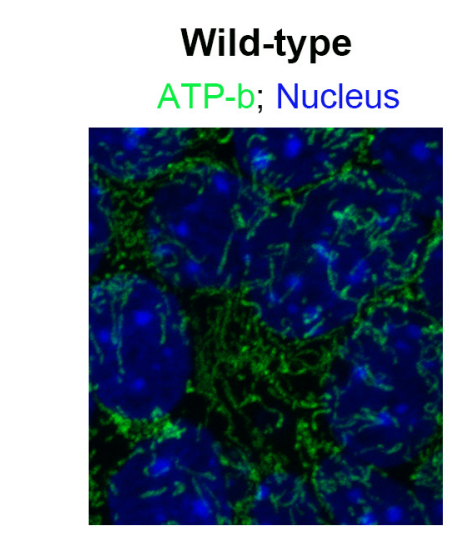

Wild-type

C

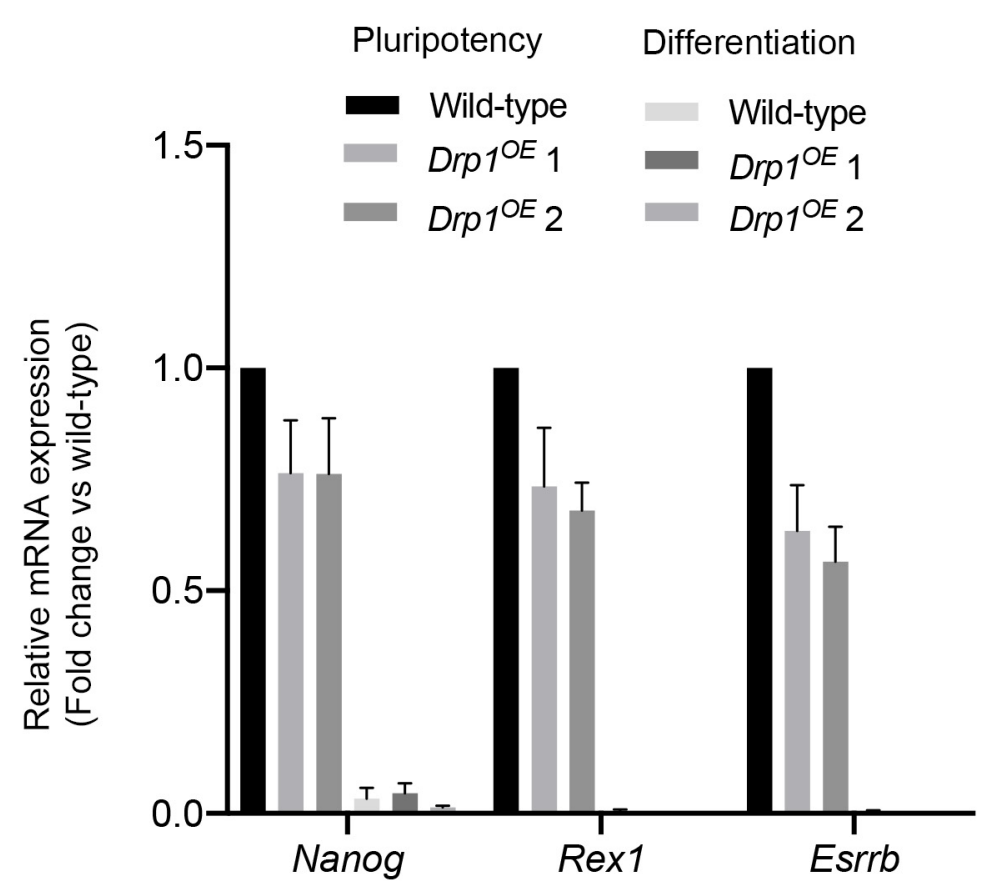

E

E ER Stress F Oxidative Stress

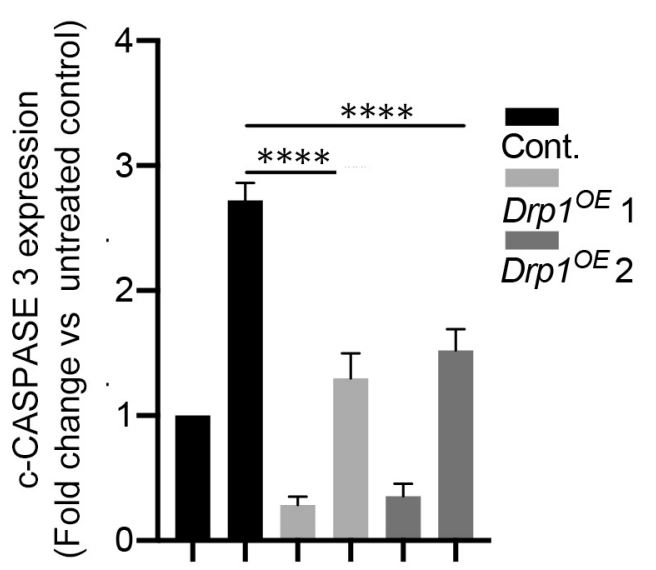

$\mathrm{Tg}$
Drp10E

ATP-b; Nucleus

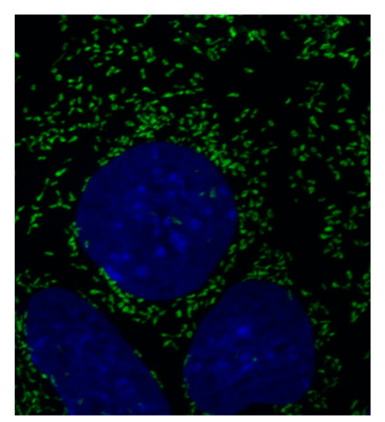

B Mitochondria circularity

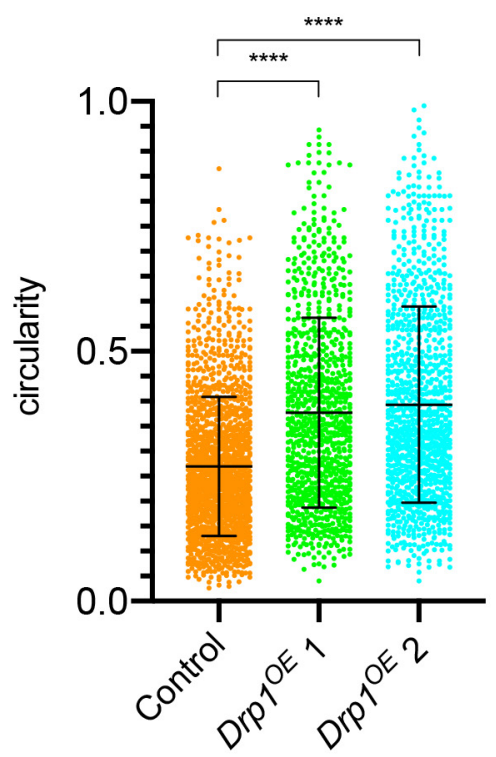

D

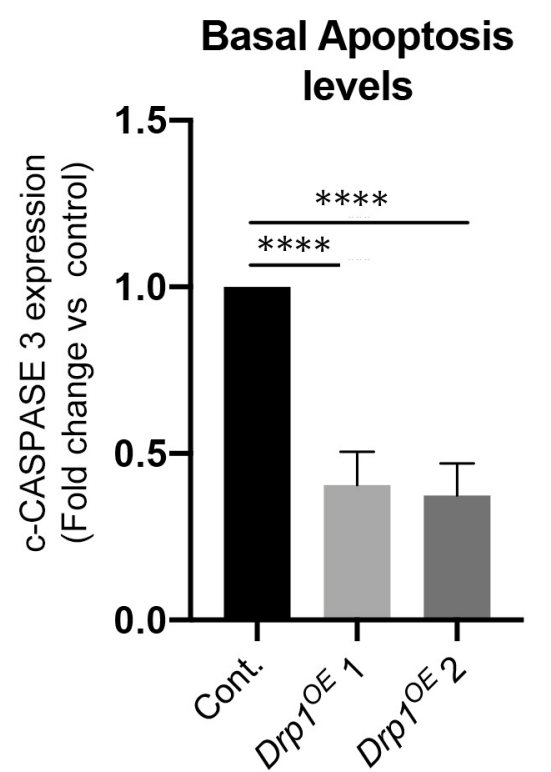

$G \quad B C L 2 / X L$ inhibition

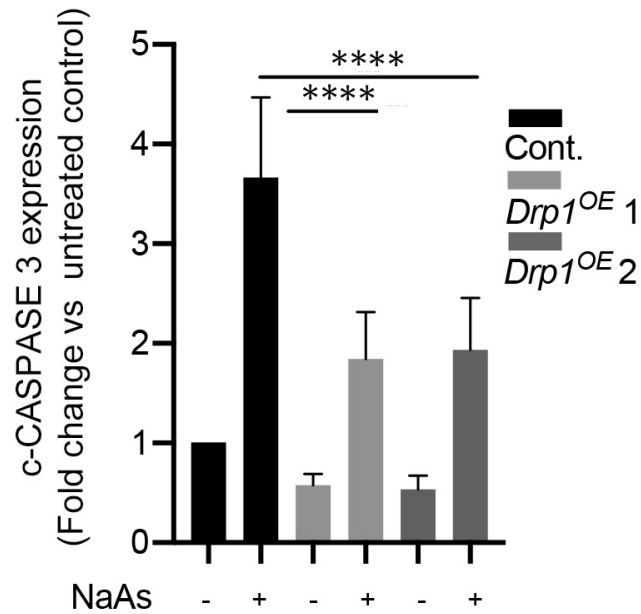

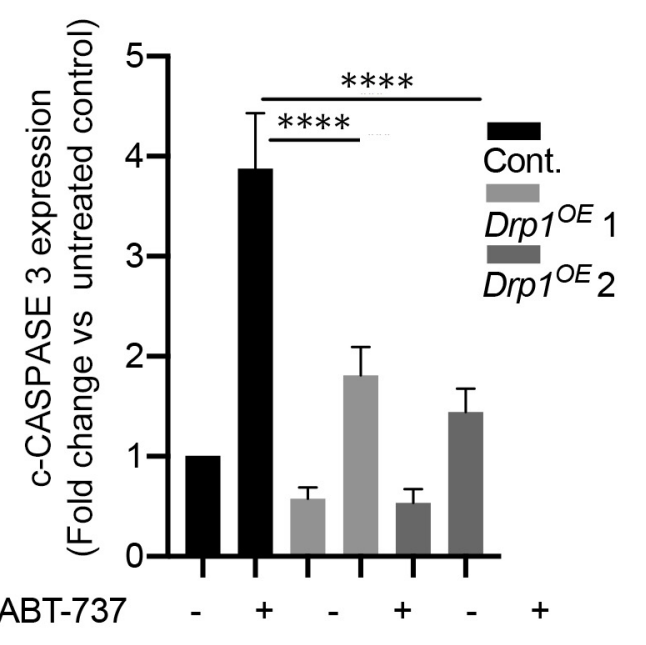




\section{Supplementary Figure 1}

A

E3.5

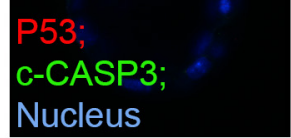

E6.5
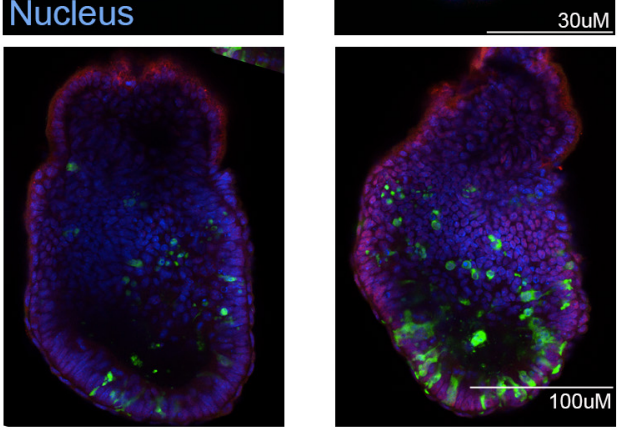

Dicer deletion in ESCs

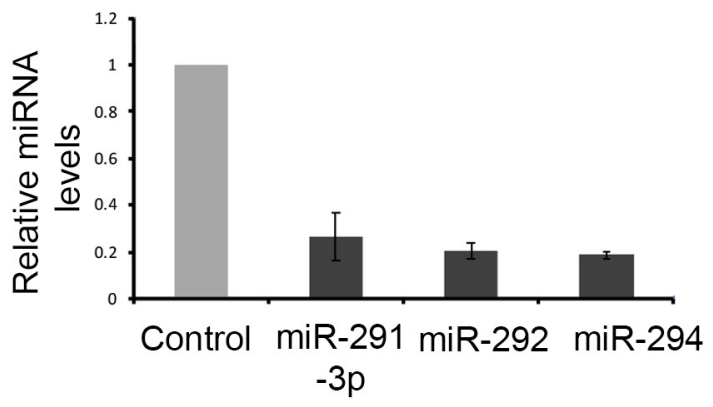

$E$

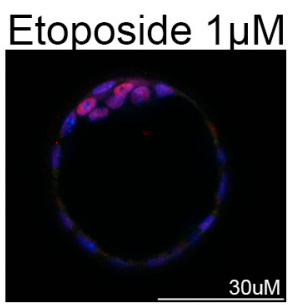

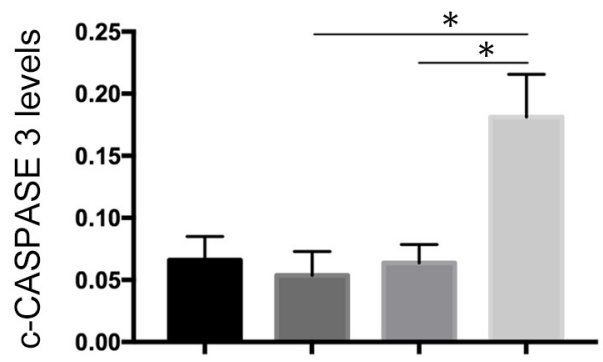

E3.5 E6.5 E3.5 E6.5 DMSO DMSO Etop. Etop.

Dicer deletion in ESCs

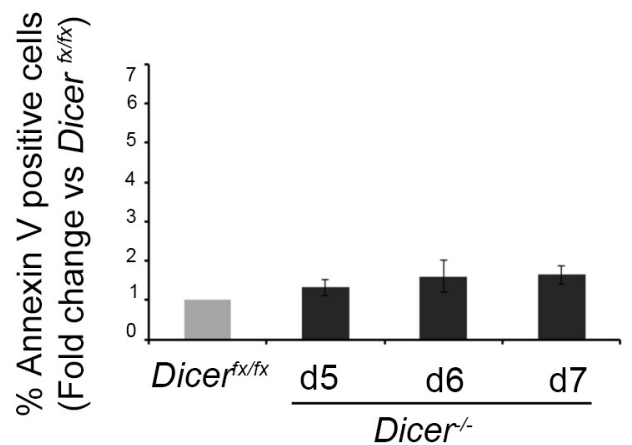

Bc/2/XL inhibition

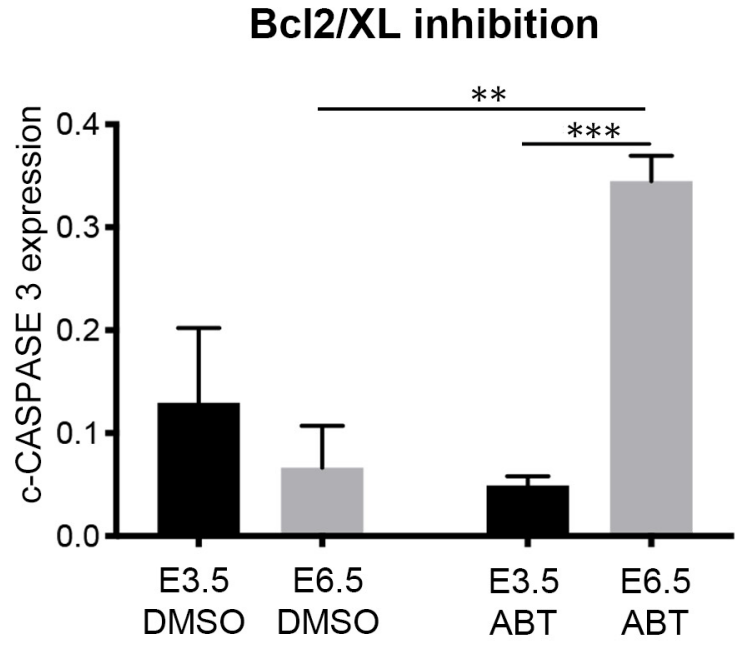

ESCs ESCs EpiSCs EpiSCs DMSO ABT DMSO ABT 
A

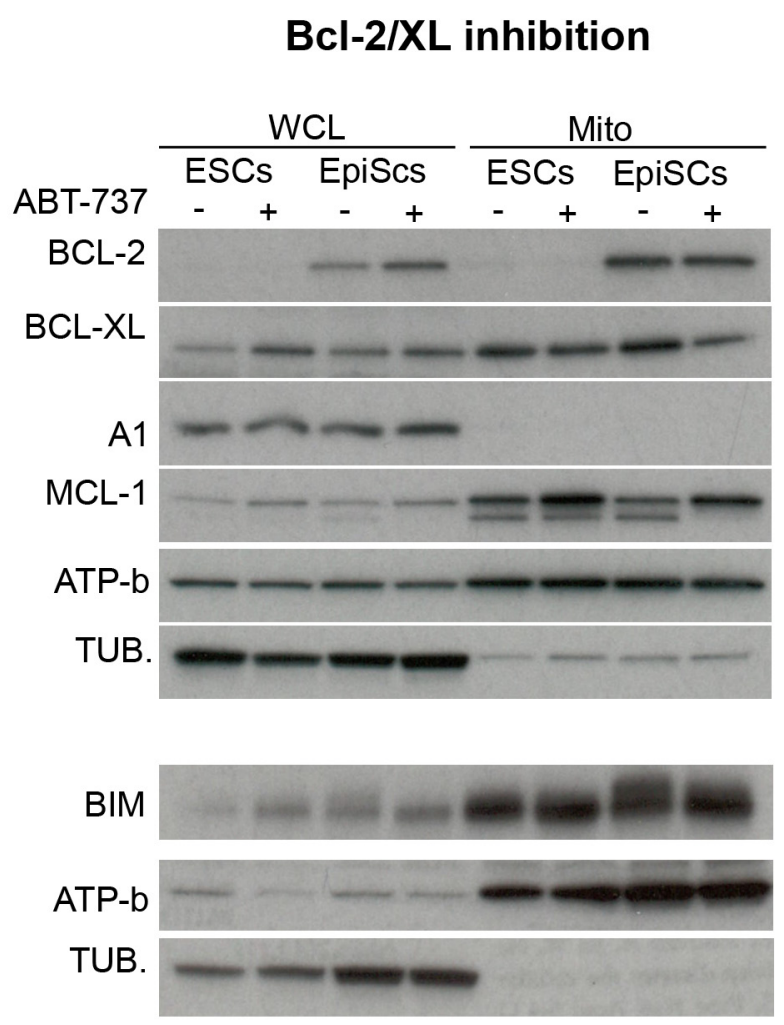

B

BCL-2
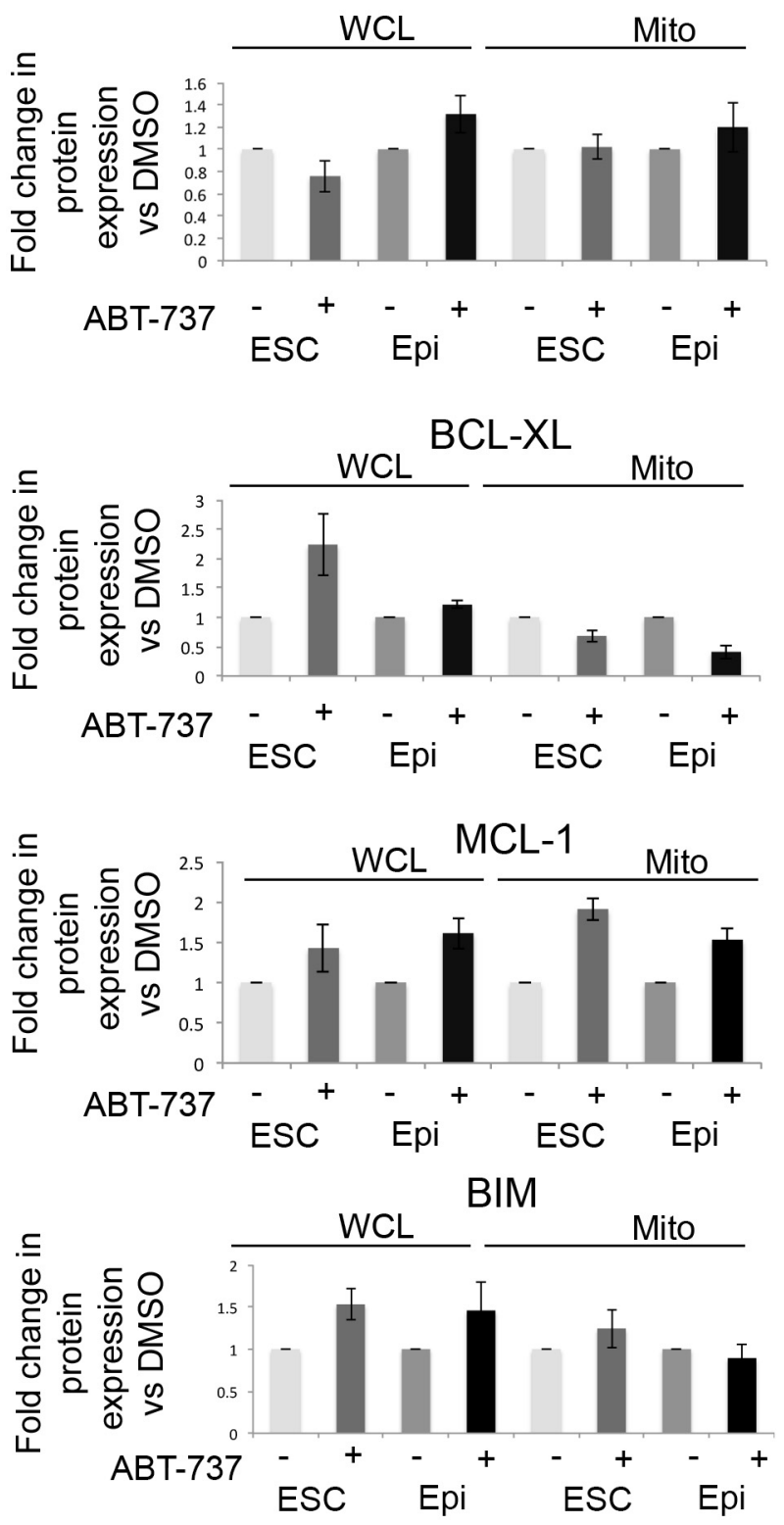
A

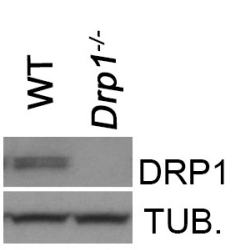

B

Glycolysis in pluripotency

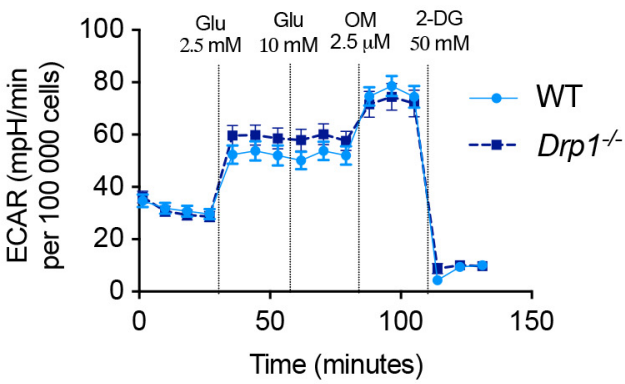

C

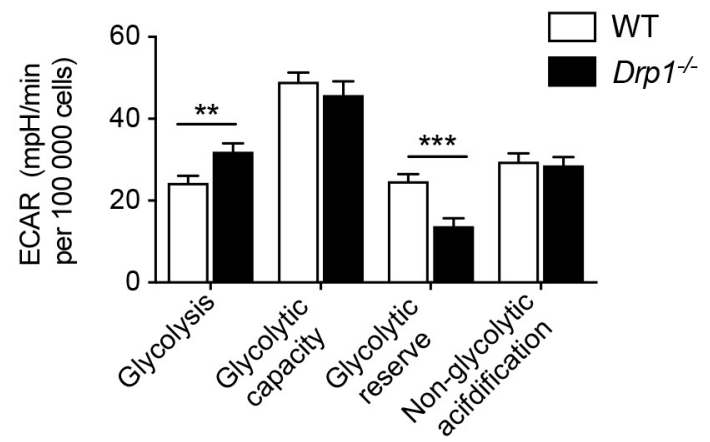

D

OXPHOS in pluripotency

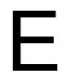

OXPHOS specific parameters
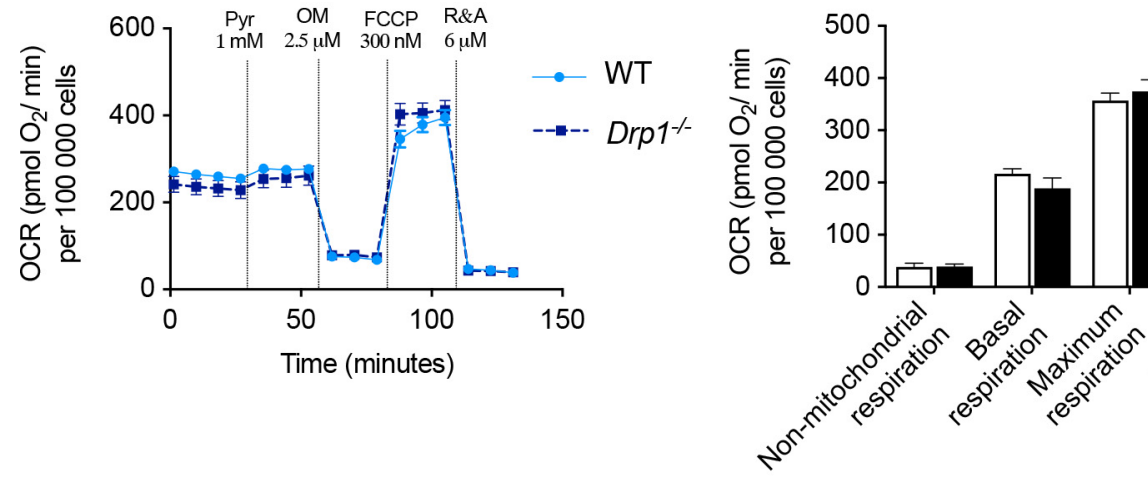

Cytosol

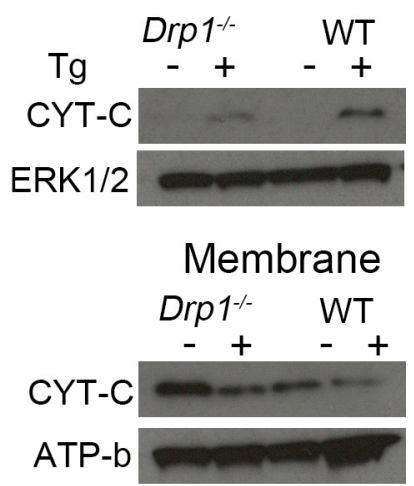

Cytosol

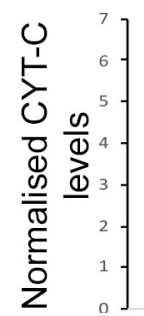

Drp 1/ $\quad$ WT
Membrane

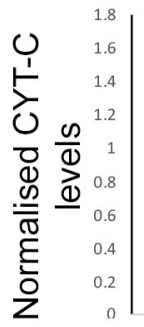

Drp1 $\quad$ WT 


\section{Supplementary Figure 4}

A

DRP1

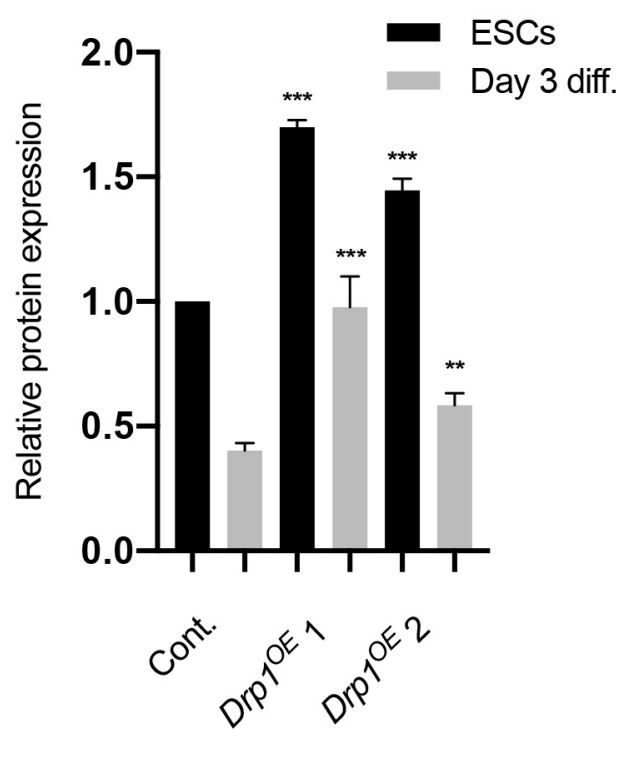

p-DRP1 (S616)

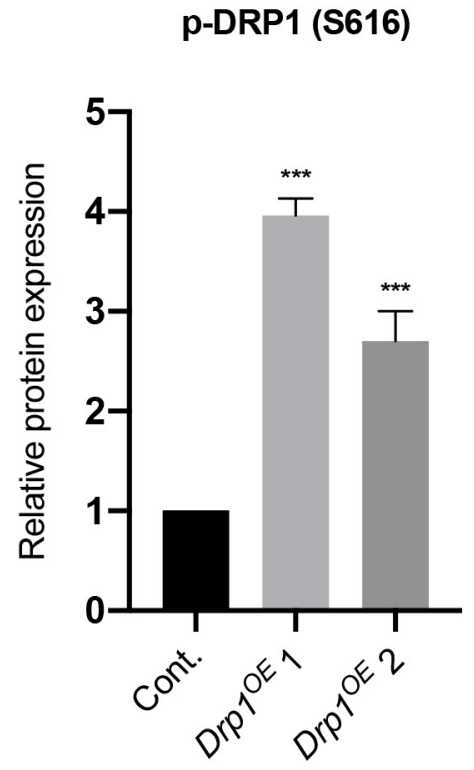

WT Drp10E

p-DRP1

TUB.

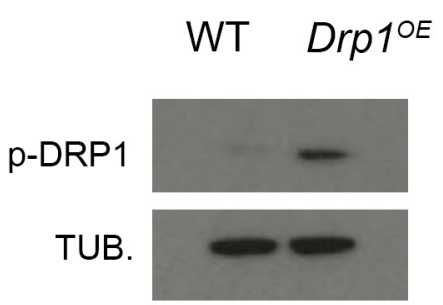

C 


\section{Supplementary Figure 5}

A

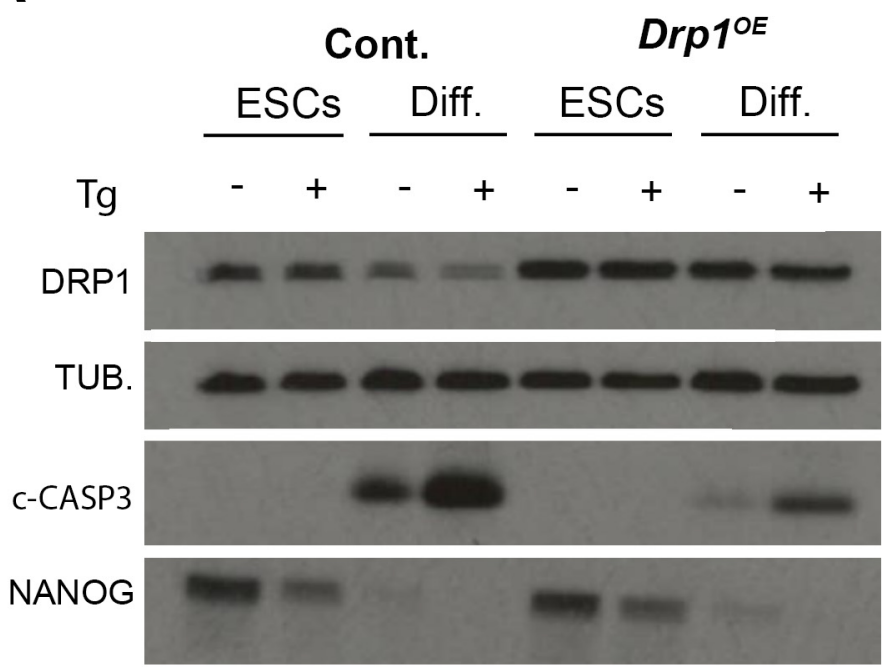

C
B

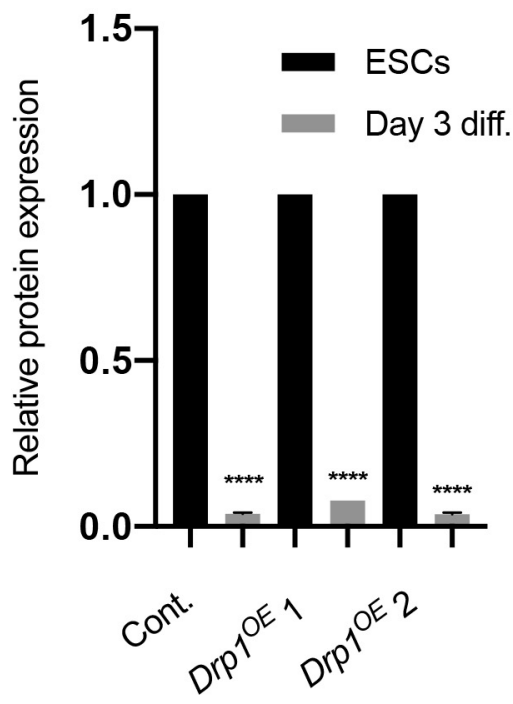

Cont. Drp1 ${ }^{O E}$

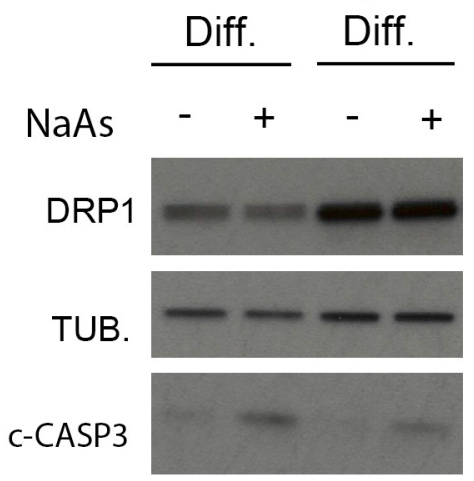

BCL2/XL inhibition

D

Cont. Drp1 ${ }^{O E}$

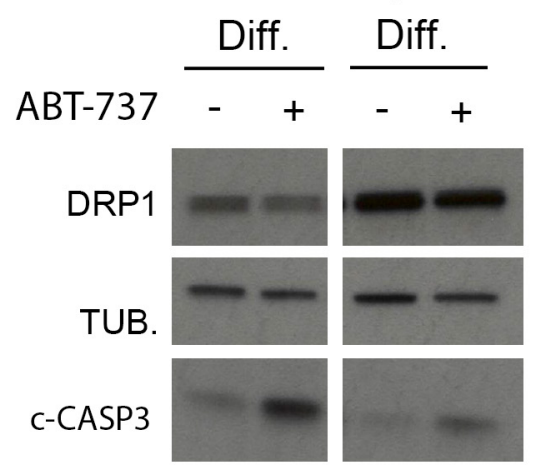

